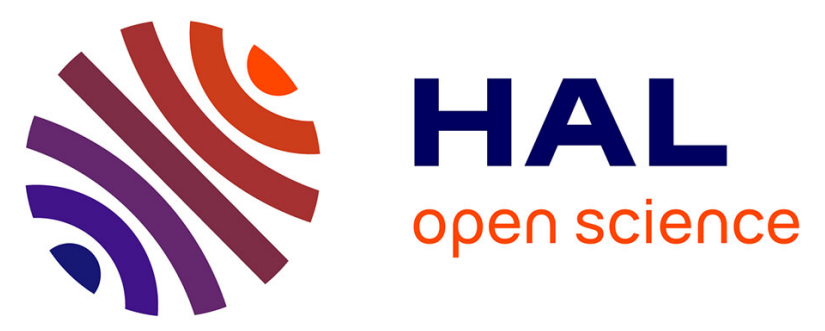

\title{
Dictyostelium lacking the single atlastin homolog Sey1 shows aberrant ER architecture, proteolytic processes and expansion of the Legionella -containing vacuole
}

Dario Hüsler, Bernhard Steiner, Amanda Welin, Bianca Striednig, A. Leoni

Leoni Swart, Virginie Molle, Hubert Hilbi, François Letourneur

\section{To cite this version:}

Dario Hüsler, Bernhard Steiner, Amanda Welin, Bianca Striednig, A. Leoni Leoni Swart, et al.. Dictyostelium lacking the single atlastin homolog Sey1 shows aberrant ER architecture, proteolytic processes and expansion of the Legionella -containing vacuole. Cellular Microbiology, 2021, 23 (5), 10.1111/cmi.13318. hal-03364992

\section{HAL Id: hal-03364992 \\ https://hal.science/hal-03364992}

Submitted on 5 Oct 2021

HAL is a multi-disciplinary open access archive for the deposit and dissemination of scientific research documents, whether they are published or not. The documents may come from teaching and research institutions in France or abroad, or from public or private research centers.
L'archive ouverte pluridisciplinaire HAL, est destinée au dépôt et à la diffusion de documents scientifiques de niveau recherche, publiés ou non, émanant des établissements d'enseignement et de recherche français ou étrangers, des laboratoires publics ou privés. 
1 Dictyostelium lacking the single atlastin homolog Sey1 shows aberrant ER architecture, 2 proteolytic processes, and expansion of the Legionella-containing vacuole 3

4 Dario Hüsler ${ }^{1}$, Bernhard Steiner ${ }^{1 \#}$, Amanda Welin ${ }^{2}$, Bianca Striednig ${ }^{1}$, A. Leoni Swart ${ }^{1}$, $5 \quad$ Virginie Molle $^{3}$, Hubert Hilbi $^{1^{*}}$, François Letourneur $^{3 *}$

6

$7{ }^{1}$ Institute of Medical Microbiology, University of Zurich, Gloriastrasse 30, 8006 Zürich,

$8 \quad$ Switzerland

$9{ }^{2}$ Division of Inflammation and Infection, Department of Biomedical and Clinical Sciences, 10 Linköping University, 58185 Linköping, Sweden

$11{ }^{3}$ Laboratory of Pathogen Host Interactions, Université de Montpellier, CNRS, INSERM, 12 Place Eugène Bataillon, Montpellier, 34095 cedex 5, France

13 Present address: " Institute for Molecular Bioscience, University of Queensland, Brisbane, 14 Queensland 4072, Australia

16 Running title: Dictyostelium atlastin controls ER architecture and Legionella replication

17 Key words: Amoeba, atlastin, Dictyostelium discoideum, endocytic pathway, large GTPase, 18 host-pathogen interaction, Legionella pneumophila, Legionnaires' disease, pathogen vacuole, 19 unfolded protein response.

20 Abbreviations: Atlastin, ATL; Icm/Dot, intracellular multiplication/defective organelle 21 trafficking; transmission electron microscopy, TEM; tunicamycin, TN; T4SS, type IV 22 secretion system; unfolded protein response, UPR.

$24 *$ *Correspondence: hilbi@imm.uzh.ch; francois.letourneur@umontpellier.fr 25 Tel.: +41(0)44 $6342650 ;+33(0) 467144731$ 


\section{ABSTRACT}

Dictyostelium discoideum Sey1 is the single ortholog of mammalian atlastin 1-3 (ATL1-3), which are large homodimeric GTPases mediating homotypic fusion of endoplasmic reticulum (ER) tubules. In this study, we generated a D. discoideum mutant strain lacking the seyl gene and found that amoebae deleted for seyl are enlarged, but grow and develop similarly to the parental strain. The $\Delta s e y 1$ mutant amoebae showed an altered ER architecture, and the tubular ER network was partially disrupted without any major consequences for other organelles or the architecture of the secretory and endocytic pathways. Macropinocytic and phagocytic functions were preserved; however, the mutant amoebae exhibited cumulative defects in lysosomal enzymes exocytosis, intracellular proteolysis, and cell motility, resulting in impaired growth on bacterial lawns. Moreover, $\Delta$ seyl mutant cells showed a constitutive activation of the unfolded protein response pathway (UPR), but they still readily adapted to moderate levels of ER stress, while unable to cope with prolonged stress. In D. discoideum Aseyl the formation of the ER-associated compartment harboring the bacterial pathogen Legionella pneumophila was also impaired. In the mutant amoebae, the ER was less efficiently recruited to the "Legionella-containing vacuole" (LCV), the expansion of the pathogen vacuole was inhibited at early stages of infection and intracellular bacterial growth was reduced. In summary, our study establishes a role of D. discoideum Sey1 in ER architecture, proteolysis, cell motility and intracellular replication of L. pneumophila.

\section{INTRODUCTION}

The endoplasmic reticulum (ER) is a pleiomorphic organelle composed of perinuclear membrane sheets and interconnected tubules extending towards the cell periphery (Shibata $e t$ al., 2006, English et al., 2009). This tubular network undergoes constant remodeling by 
51 homotypic fusion of ER tubules. Numerous proteins are required to form and maintain this dynamic ER network (Shibata et al., 2009). Among them, the membrane-bound large GTPase atlastin (ATL) has been shown to mediate the homotypic fusion between tubules, ensuing the formation of three-way junctions and subsequent extensive reticulation of the ER network (Hu et al., 2009, Orso et al., 2009). In mammals, three ATL paralogs (ATL1-3) are selectively produced in different tissues. ATLs are highly conserved during evolution with only one single ortholog produced in yeast, plants or the amoeba Dictyostelium discoideum (named Sey1 in these different organisms) (Chen et al., 2011, Anwar et al., 2012, Zhang et al., 2013, Steiner et al., 2017). ATLs share a similar domain organization comprised of an Nterminal GTPase and a three-helix bundle (3HB), followed by two closely spaced predicted transmembrane (TM) segments and a C-terminal tail (CT). The role of ATLs in ER network formation is emphasized by the observation that mutations in the ATL genes were detected in the human neurological disorders hereditary spastic paraplegia (HSP) and hereditary sensory neuropathy (HSN), which are characterized by severe ER morphological defects (Salinas et al., 2008, Guelly et al., 2011, Fink, 2013, Fischer et al., 2014, O'Donnell et al., 2018). Furthermore, ATL gene deletion and overexpression of dominant-negative mutants result in 67 defects of ER network formation (Rismanchi et al., 2008, Hu et al., 2009, Wang et al., 2016, 68 Zhao et al., 2016).

69 Structural and biochemical studies indicate that upon GTP binding, the GTPase domains of 70 ATL from two distinct ER tubules can form trans-homodimers, thus promoting membrane 71 contact and fusion (Bian et al., 2011, Byrnes et al., 2011). The fusogenic properties of ATLs 72 have been molecularly detailed by in vitro membrane fusion studies (Orso et al., 2009, Bian et 73 al., 2011, Anwar et al., 2012, Kim et al., 2017) and crystal structure analysis (Bian et al., 74 2011, Byrnes et al., 2011, Liu et al., 2012, Byrnes et al., 2013, Liu et al., 2015). In addition to 75 ER dynamics, ATLs contribute to other cellular processes including lipid droplet biogenesis 
76 (Klemm et al., 2013), mitochondrial lipid exchange (Voss et al., 2012), membrane tethering 77 (Krols et al., 2018, Niu et al., 2019), endosomal transport (Stefano et al., 2015), inner nuclear

membrane protein insertion (Pawar et al., 2017), and selective autophagy (Liang et al., 2018, Chen et al., 2019).

Owing to numerous convenient genetic and biochemical tools, the amoeba Dictyostelium discoideum has been widely used as a model organism to study eukaryotic cell biology and host-pathogen interactions (Cardenal-Munoz et al., 2017, Swart et al., 2018). The phagocytic amoebae can engulf and digest bacteria as nutritional sources. However, some pathogenic bacteria avoid the degradative functions of $D$. discoideum and replicate inside this host cell. For instance, Legionella pneumophila, the causative agent of a severe pneumonia in humans termed Legionnaires' disease, induces the formation of a replication-permissive membranebound compartment called "Legionella-containing vacuole" (LCV) in the amoeba (Swart et al., 2018).

The LCV biogenesis mechanism is comparable in human macrophages and amoeba (Gomez-Valero et al., 2011, Al-Quadan et al., 2012), and relies on the delivery of more than 300 different "effector" proteins into host cells by the bacterial Icm/Dot (Intracellular multiplication/defective organelle trafficking) type IV secretion system (T4SS). These effectors target and control key host cell processes including vesicular trafficking in the endocytic, secretory and retrograde pathways, lipid metabolism, translation, transcription, and apoptosis (Isberg et al., 2009, Asrat et al., 2014, Finsel et al., 2015, Personnic et al., 2016, Barlocher et al., 2017, Steiner et al., 2018a, Swart et al., 2020a). The hijacking of host cell functions notably leads to the recruitment of ER-derived vesicles (Kagan et al., 2004), as well as rough ER to LCVs (Swanson et al., 1995, Robinson et al., 2006, Weber et al., 2014b, Weber et al., 2018). The ER associating with LCVs might provide structural elements for 
100 pathogen vacuole formation and expansion, nutrients for intracellular growth, and/or 101 camouflage from host cell defenses (Sherwood et al., 2016).

102 ATL3 and Sey1 have been identified by proteomics in intact LCVs purified from $L$. 103 pneumophila-infected macrophages or Dictyostelium, respectively (Hoffmann et al., 2014). 104 Recently, we validated the presence of ATL3/Sey1 on LCVs, and we revealed a functional 105 role of this large GTPase for LCV formation (Steiner et al., 2017). However, these studies 106 were limited to an analysis of infected D. discoideum or isolated LCVs upon overproduction 107 of a dominant negative Sey1 mutant, hampering further comprehensive studies. In order to 108 assess the role of Sey1 for cellular processes and host-pathogen interactions, we adopted a 109 genomic deletion strategy. In the present study, we uncovered the morphological and 110 functional consequences of Sey1 genetic inactivation in Dictyostelium and further detailed 111 Sey1 function(s) in the formation and expansion of the Legionella replicative vacuole.

\section{RESULTS}

\section{$114 \quad 2.1$ Construction and basic characterization of $\boldsymbol{D}$. discoideum $\Delta$ sey1 cells}

115 The only atlastin protein in D. discoideum is termed Sey1 (Steiner et al., 2017). To analyze

116 the function of Sey1, the corresponding gene was disrupted by insertion of the blasticidin 117 selection marker by double homologous recombination (Figure 1a). This insertion left only a 118 residual protein of 148 amino acids without any Seyl functional domains. Several 119 independent clones were analyzed and displayed identical phenotypes, which were 120 complemented by transfection with the seyl coding sequence (see hereafter and data not 121 shown).

122 When attached to standard plastic cell culture dishes, D. discoideum lacking Seyl ( $\Delta$ seyl) 123 showed heterogeneously enlarged cell-surface size compared to Ax3 parental cells (2.2 times 124 the normal median size; Figures $1 \mathrm{~b}$ and 1c). Electric current exclusion measurements (CASY 
125 analyzer) further confirmed that $\Delta$ seyl cells had a greater diameter heterogeneity than Ax3

126 cells (mean diameters of $10.24 \mu \mathrm{m}$ or $9.51 \mu \mathrm{m}$, respectively; Figure $1 \mathrm{~d}$ ). The seyl mutant

127 amoebae were also frequently polynucleated (Figure 1e), suggesting a cytokinesis defect as

128 described in fission yeast mutants affecting the cortical ER structure (Zhang et al., 2010).

129 Moreover, the relative growth rate of the $\Delta$ seyl strain was slightly reduced, and a plateau

130 phase was reached at a lower cell density compared to Ax3 cells (Figure 1f). Upon starvation,

131 aggregates and fruiting bodies were observed with genuine timing (Figure 1g). In summary,

132 D. discoideum deleted for the seyl gene is viable and, compared to the parental strain, slightly

133 enlarged and more frequently polynucleated, but develops largely normally. Moreover, the

$134 \Delta$ seyl mutant strain grows slightly slower in axenic liquid medium.

\subsection{D. discoideum lacking Sey1 shows aberrant ER morphology and dynamics}

137 Since atlastin GTPases participate in the formation of the tubular ER network in other 138 organisms, we examined the ER morphology in D. discoideum $\Delta$ seyl cells by confocal 139 microscopy. In Ax3 parental cells, the ER-resident enzyme protein disulfide isomerase (PDI)

140 localized both at the nuclear periphery and at interconnected ER tubules, which appeared to

141 be extended throughout the entire cell towards the cell periphery (Figure 2a). In contrast, most

$142 \Delta$ seyl cells showed clumps of PDI positive tubules at the cell periphery and a reduced

143 interconnected ER tubule network. This altered ER morphology was identical in fixed and

144 live cells producing the ER-resident reporter protein calnexin A-GFP (CnxA-GFP) (Figures

$1452 \mathrm{~b}$ and 2c). Furthermore, ectopic production of GFP-Sey1 but not GFP-Sey1_K154A

146 (GTPase defective mutant) in $\Delta$ seyl cells restored a normal ER morphology (Figure S1a),

147 indicating that the GTPase activity of Sey1 is required to form and maintain a native ER

148 network. 
149 Moreover, the ER-shaping protein reticulon (Rtnlc in D. discoideum), an interactor of 150 Sey1, localized to ER tubules (but not to the perinuclear ER) in Ax3 and $\Delta$ seyl cells upon 151 overproduction of an N-terminal GFP fusion protein, GFP-Rtnlc (Figure S1b). The ER152 resident SNARE (soluble NSF attachment receptor) proteins Sec22b, Ufe1, and Use1 153 localized to ER tubules as well as the perinuclear ER in Ax3 and $\Delta$ seyl cells upon 154 overproduction of the corresponding GFP fusion proteins (Figure S2). Taken together, Sey1 is 155 required to establish normal ER architecture, but appears dispensable for the correct 156 localization of Rtnlc and ER SNARE proteins.

157 The ER network is a highly dynamic structure, wherein tubules are constantly branching 158 and fusing with each other (Schwarz et al., 2016). To determine whether the absence of Sey1 159 might affect ER tubule dynamics, D. discoideum cells producing GFP-calnexin were analyzed 160 by time-lapse video microscopy. While in the parental Ax3 strain, GFP-calnexin-positive ER 161 tubules showed vivid dynamics, in live $\Delta$ seyl cells, GFP-calnexin-labeled nodular clumps 162 were observed, which were mostly static. However, some faintly labeled ER tubules localized 163 between the nucleus and the cell periphery and showed dynamics similar to that of Ax3 cells 164 (Figure 2c, and Movies S1 and S2). These results indicate that tubule fusion and branching 165 still occur in $\Delta$ sey 1 cells, however, to a much smaller extent.

166 Additional fluorescence microscopy analysis revealed that the morphology and distribution 167 of other intracellular compartments was not altered in $\Delta s e y 1$ cells, and the architecture and 168 dynamics of the microtubule cytoskeleton was fully conserved in the mutant cells (Figure S3, 169 and Movies S3 and S4). Most importantly with regard to the functional analyses presented 170 hereafter, the overall structure of endocytic compartments appeared unaffected in $D$. 171 discoideum $\Delta$ seyl (Figure S3), and the subcellular distributions of PtdIns(3)P and PtdIns(4)P, 172 hallmark phosphoinositide lipids of the endocytic and secretory pathway, respectively, 173 appeared normal (Figures S4a and S4b). These conclusions were further supported by 
174 transmission electron microscopy (TEM) analysis which revealed no additional ultrastructural

175 defects in $\Delta$ seyl cells besides the aberrant ER morphology observed by optical microscopy

176 (Figure 2d). Taken together, the D. discoideum $\Delta$ seyl strain exhibits an aberrant ER tubule

177 network characterized by clumps of PDI- and calnexin-positive tubules, but otherwise appears

178 largely normal regarding cell organelle and cytoskeleton architecture and dynamics.

179

$180 \quad 2.3 \mathrm{D}$. discoideum $\Delta$ sey1 cells are impaired for growth on bacterial lawns

181 The reduced growth rate of $\Delta s e y l$ cells in axenic liquid medium prompted us to examine their

182 endocytic capacities. $D$. discoideum feeds on liquid medium by macropinocytosis. To

183 evaluate this process, cells were incubated with fluorescent dextran for different periods of

184 time, and uptake was measured by flow cytometry analysis. Dextran uptake occurred at a

185 normal rate in $\Delta$ seyl cells, excluding that nutrient absorption is the growth-limiting step

186 (Figure 3a). In D. discoideum, lysosomes mature into post-lysosomes, which eventually fuse

187 with the plasma membrane and release their contents to the outside of the cells by exocytosis.

188 In $\Delta$ seyl cells, the rate of exocytosis of fluorescent dextran showed a moderate but significant

189 increase $(51.3 \%$ of initial fluorescence retained after a chase period of $80 \mathrm{~min}$ in $\Delta$ seyl cells

190 compared to $66.1 \%$ in Ax3 cells) (Figure 3b).

191 D. discoideum can ingest other sources of nutrients (e.g. bacteria) by phagocytosis.

192 Surprisingly, $\Delta$ seyl cells could hardly grow on lawns of live (or even heat-killed) Klebsiella

193 pneumoniae, unless the mutant strain was complemented by plasmid-borne GFP-Sey1 and

194 myc-tagged Sey1 (Figures 3c and S5a). In contrast, the ectopic production of GFP-Sey1 in the

195 parental Ax3 strain did not affect its growth on a $K$. pneumoniae lawn. The growth defect of

$196 \Delta s e y 1$ was also observed with other bacterial strains, including Escherichia coli $\mathrm{B} / \mathrm{r}$ and to a

197 lesser extent with Bacillus subtilis and Micrococcus luteus (Figure 3d). Thus, the growth 
198 defect of $D$. discoideum $\Delta$ seyl occurs on lawns of different bacteria and is not restricted to 199 particular Gram-negative or Gram-positive bacteria.

200 To grow on bacteria, D. discoideum has to bind, internalize, kill, and finally digest the 201 bacterial cells. Interestingly, sseyl cells showed normal uptake of bacteria (K. pneumoniae) 202 and fluorescent beads (Figures 3e and 3f), excluding any major defects in particle 203 binding/recognition and uptake. Furthermore, identical bacteria killing efficiencies were 204 observed for Ax3 and $\Delta$ seyl cells (Figure 3g). In summary, compared to the parental strain, $205 \Delta$ seyl cells show similar capacities for macropinocytosis, phagocytosis and exocytosis, but 206 are severely impaired for growth on lawns formed by a range of bacteria. Together these 207 results suggest that defective digestive functions might account for the growth defects of $208 \Delta$ seyl cells.

\subsection{Lysosomal enzymes are hyper-secreted by $\Delta$ sey 1 cells}

211 To evaluate the digestion of bacteria by $D$. discoideum $\Delta$ seyl, we first measured the activity 212 of lysozymes, a family of enzymes responsible for the degradation of bacterial cell wall 213 peptidoglycans (Lamrabet et al., 2020). Two complementary assays revealed that $\Delta$ seyl cells 214 exhibited only $75 \%$ of the intracellular activity of lysozymes measured in Ax3 cells (Figure 215 4a). Since exocytosis is increased in $\Delta$ seyl cells (Figure 3b), we hypothesized that lysosomal 216 enzymes might be hyper-secreted, consequently decreasing their intracellular pool and 217 impairing degradative functions of the lysosomes. To test this hypothesis, we measured the 218 enzymatic activities of several lysosomal enzymes in cell and culture medium fractions 219 separated after cultivation for $24 \mathrm{~h}$. A higher percentage of $\alpha$-mannosidase, cathepsin D 220 (CtsD), and $\mathrm{N}$-acetyl-glucosaminidase was secreted by $\Delta$ sey 1 compared to Ax3 cells, while 221 the secretion of acid phosphatase was the same (Figures $4 \mathrm{~b}$ and S5b). This secretion 222 phenotype was largely reversed by the ectopic production of GFP-Sey1 (Figure 4b). 
In addition, we noticed that hypersecretion of lysosomal enzymes was associated with 224 reduced total activities of $\alpha$-mannosidase and CtsD inside the cells (Figure S5c). This 225 decrease was caused by lower amounts of cellular lysosomal enzymes and not by reduction of 226 their hydrolytic capacities when intracellularly retained. This was verified for CtsD by 227 Western blot of whole cell lysates with an anti-CtsD antibody. Accordingly, in $\Delta s e y l$ cells, a 228 reduced amount of CtsD was observed in cell lysates, and this was partially compensated by 229 ectopic production of GFP-Sey1 (Figures 4c and 4d).

230 We also noticed that the apparent molecular weight of CtsD in $\Delta$ seyl cells corresponds to 231 the mature form of this enzyme (Figure 4c) (Journet et al., 1999), suggesting that post232 translational protein modifications occur normally in $\Delta s e y l$ cells. This was further confirmed 233 by the analysis of a highly glycosylated reporter protein, contact-site A (CsA) fused to 234 transmembrane and cytoplasmic domains of SibA (Froquet et al., 2012). This CsA-SibA 235 chimeric protein showed the expected $\mathrm{N}$ - and O-glycosylation patterns in $\Delta$ seyl cells, 236 reflecting a preserved genuine glycosylation process (Figure S5d).

237 Moreover, the acidification of endocytic compartments, which is required for optimal 238 function of lysosomal enzymes, occurred at normal rates in $\Delta$ seyl cells, excluding any 239 enzymatic activity defects due to non-optimal $\mathrm{pH}$ of degradative compartments (Figure S5e). 240 Finally, total protein degradation in phagosomes was directly assessed by measuring 241 proteolysis of DQ Green-BSA-coated beads at $1 \mathrm{~h}$ after cellular uptake. This assay revealed a $24210 \%$ reduction of proteolysis in $\Delta$ seyl cells (Figure $4 \mathrm{e}$ ). Together, these observations indicate 243 that the intracellular retention of lysosomal enzymes is impaired in $\Delta$ seyl cells, consequently 244 likely reducing the hydrolytic processing of nutrients required for optimal growth of the 245 amoeba. 
248 Upon growth on bacterial lawns, D. discoideum cells leave "grazed" regions and move 249 towards more nutritive areas. To assess whether in addition to the impaired lysosomal 250 degradation, a reduced cell motility could contribute to the growth defect of $\Delta$ seyl cells, 251 random movement of individual cells seeded on a glass substrate in culture medium was 252 recorded by time-lapse confocal microscopy (Figure 5a). Mutant $\Delta$ seyl cells showed a 253 markedly reduced motility with a maximum distance from the initial position (D-max) of 23.0 $254 \pm 8.2 \mu \mathrm{m}$ compared to $97.4 \pm 21.4 \mu \mathrm{m}$ for the Ax3 cells (Figure $5 \mathrm{~b}$ ). The ectopic production 255 of GFP-Sey1 in $\Delta$ sey 1 cells partially restored motility (D-max $=68.6 \pm 22.5 \mu \mathrm{m})$, in contrast 256 to the GTPase defective mutant GFP-Sey1_K154A, which had almost no effect (D-max = $25736.6 \pm 15.9 \mu \mathrm{m})$. Moreover, while GFP-Sey1 did not affect the motility of Ax3 cells, the 258 GFP-Sey1_K154A mutant protein strongly inhibited their motility (D-max $=51.5 \pm 19.0 \mu \mathrm{m})$, 259 indicating that the GTPase activity and/or dimerization of Sey1 is required for protein 260 function, and consequently, cell motility. Since $\Delta$ seyl cells exhibited no major alteration in 261 the organization of F-actin cellular protrusions (Figure S4c), other key players in cellular 262 motility are likely affected by Sey1 deletion. In summary, dependent on its GTPase activity, 263 Sey1 regulates the motility of $D$. discoideum.

\subsection{Sey1 contributes to ER homeostasis in stressed cells}

266 Atlastins in mammalian cells and RDH3 in plants have been shown to participate in the ER 267 stress response (Lai et al., 2014, Liang et al., 2018, Chen et al., 2019, Niu et al., 2019). To 268 test whether Seyl adopts similar functions in D. discoideum, $\Delta$ seyl cells were incubated with 269 tunicamycin (TN), a compound which induces ER stress and triggers the unfolded protein 270 response (UPR) pathway (Dominguez-Martin et al., 2018a, Dominguez-Martin et al., 2018b). 271 After $24 \mathrm{~h}$ of treatment the viability of $\Delta s e y 1$ was only slightly reduced compared to Ax 3 cells 272 (Figure 6a). However, this difference was dramatically amplified after $48 \mathrm{~h}$ and $72 \mathrm{~h}$. After 48 
$273 \mathrm{~h}$, only $21.5 \% \Delta$ seyl cells were alive compared to $69.2 \% \mathrm{Ax} 3$ cells, and at $72 \mathrm{~h}$ the difference 274 was also more than 3 -fold. Phase contrast microscopy observations at $48 \mathrm{~h}$ further confirmed 275 these results (Figure 6b).

276 Interestingly, the viability difference between $\Delta$ seyl and the parental Ax3 strain was 277 dependent on the TN dose (Figure 6c). The viability of $\Delta$ seyl cells dropped to $45.9 \%$ at 125 $278 \mathrm{ng} / \mathrm{mL}$ TN and reached a minimum of $20.2 \%$ viable cells at $1 \mu \mathrm{g} / \mathrm{mL} \mathrm{TN}$. In contrast, Ax3 279 parental cells as well as $\Delta s e y l$ cell ectopically producing RFP-Seyl showed only a mild 280 reduction in viability $(67.7 \%$ and $61.1 \%$ viable cells, respectively) even at the highest 281 concentration of TN tested $(1.5 \mu \mathrm{g} / \mathrm{mL})$. Thus, $\Delta$ seyl cells manage to readily adapt to short282 term ER stress, but exhibit strikingly reduced capacities to cope with prolonged ER stress.

283 To restore cellular homeostasis upon ER stress, cells increase protein degradation rates by 284 several mechanisms, including the ubiquitin-dependent ERAD (ER-associated degradation) 285 pathway and macroautophagy (hereafter referred to as autophagy) (Almanza et al., 2019). To 286 assess a role for Sey1 in ERAD, we first tested the production of CdcD (ERAD protein 287 homologous to human $\mathrm{VCP} / \mathrm{p} 97$ and yeast $\mathrm{Cdc} 48$ ) in response to TN (Figure 6d and 6e). In 288 agreement with published results (Dominguez-Martin et al., 2018b), CdcD production 289 increased in Ax3 cells upon addition of $\mathrm{TN}$ for $24 \mathrm{~h}$. A similar induction was observed in $290 \Delta$ seyl cells; however, the basal production of $\mathrm{CdcD}$ in untreated cells was higher in $\Delta$ seyl 291 than in Ax3 cells. This result might reflect a constitutive activation of the ERAD pathway in 292 mutant cells. In contrast, the basal production of PDI, a multifunctional ER-resident redox 293 chaperone, was not enhanced in resting $\Delta$ seyl cell, whereas TN treatment resulted in identical 294 induction of PDI production in $\Delta s e y 1$ and Ax3 cells, as expected for a treatment known to 295 augment protein folding capacities (Figure 6d and 6e). In summary, compared to the parental 296 Ax3 strain $\Delta$ sey 1 cells are more sensitive to ER stress. 
$298 \quad 2.7$ Intracellular replication and killing of $L$. pneumophila in $\Delta s e y 1$ cells

299 Intracellular replication of L. pneumophila occurs in ER-associated LCVs and implicates 300 Sey1 (Steiner et al., 2017). To test intracellular growth of L. pneumophila in D. discoideum $301 \Delta$ seyl cells, we used mCherry-producing bacteria and quantified fluorescence intensity (RFU) 302 as well as the number of colony-forming units (CFU) (Figures 7a and S6a). In both assays, 303 wild-type L. pneumophila showed a severe growth defect upon replication in $\Delta$ seyl cells. On 304 the other hand, the L. pneumophila $\Delta i c m T$ mutant strain was unable to replicate in the $D$. 305 discoideum Ax3 or $\Delta s e y 1$ strain (Figure 7a), and killing (or survival) of $\Delta i c m T$ bacteria was 306 not different in D. discoideum $\Delta$ seyl compared to the parental strain Ax3 (Figure 7b). Taken 307 together, L. pneumophila replicates less efficiently in $\Delta s e y l$ compared to the parental strain, 308 and hence, Sey1 promotes intracellular replication of L. pneumophila.

\subsection{Recruitment of rough ER to LCVs is impaired in D. discoideum $\Delta$ sey1 cells}

311 Given the various defects of $D$. discoideum $\Delta$ seyl cells in ER morphology and dynamics, we 312 sought to assess LCV formation in the mutant amoebae. In order to obtain high-resolution 313 images of LCVs in D. discoideum Ax3 and $\Delta$ seyl cells, we used regular TEM (Figure 7c). 314 Upon infection with wild-type L. pneumophila, rough ER was observed at $2 \mathrm{~h}$ p.i. and $8 \mathrm{~h}$ p.i. 315 around LCVs in D. discoideum Ax3 and in $\Delta$ seyl cells producing plasmid-borne GFP-Sey1, 316 but not in $\Delta s e y 1$ mutant cells. No ER accumulated around vacuoles harboring the avirulent $L$. 317 pneumophila $\Delta i c m T$ in any of these $D$. discoideum strains at any time, as has been observed 318 previously with amoebae producing dominant-negative GFP-Sey1_K154A (Steiner et al., 319 2018b). In summary, in agreement with the severe defect of $\Delta$ seyl cells regarding ER 320 morphology and dynamics, LCVs harboring wild-type L. pneumophila are not decorated with 321 rough ER in the mutant amoebae. 
323

324

325

326

327

328

329

\subsection{PtdIns(4)P-positive LCVs in D. discoideum $\Delta$ sey 1 acquire ER less robustly}

Next, we assessed LCV formation by confocal microscopy using dually labeled $D$. discoideum Ax3 or $\Delta$ seyl producing the PtdIns(4)P/LCV probe P4C-mCherry and the ER marker calnexin-GFP. Compared to the uninfected dually labeled Ax3 strain, in $\Delta s e y 1$ cells the ER was disorganized, fragmented and showed a vesicular organization (Figures $2 b$ and S6b). This defective ER morphology was retained in L. pneumophila-infected $\Delta s e y 1$ cells and was particularly striking at early time points of infection (up to 8 h p.i.; Figure 8).

In general, LCVs formed by wild-type L. pneumophila in the $\Delta$ sey 1 mutant accumulated less calnexin-positive ER than LCVs in the parental strain Ax3, in particular at early time points during infection ( 2 h p.i.) (Figure $8 \mathrm{a})$. These fluorescence microscopy observations validate the TEM data (Figure 7c). In the $\Delta$ seyl as well as in the Ax3 parental strain, the ER localized to LCVs not homogenously but in a rather patchy manner. Moreover, the PtdIns(4)P-positive limiting LCV membrane was formed in $\Delta$ seyl cells as well as the parental strain, and remained clearly visible throughout the infection $(2 \mathrm{~h}-48 \mathrm{~h}$ p.i.) (Figures $8, \mathrm{~S} 6 \mathrm{c}$, and S6d).

To quantify the recruitment of calnexin to PtdIns(4)P-positive LCVs in an unbiased and high-throughput manner, we used imaging flow cytometry (IFC). To this end, we infected the dually labeled $D$. discoideum strain producing P4C-mCherry and calnexin-GFP with mPlumproducing L. pneumophila (Figure 8b). The IFC colocalization score (see Materials and

342 Methods) for the acquisition of calnexin-GFP to LCVs was then assessed. The calnexin-GFP

343 colocalization score on LCVs formed by wild-type L. pneumophila increased during early

344 time points of infection ( 1 h -6 h p.i.) (Figure $8 b$ ), and was significantly lower in amoebae

345 lacking Sey1. The calnexin-GFP colocalization score on LCVs decreased at later time-points

346 (24 h - 48 h p.i.; Figure S6e). Finally, calnexin-GFP did not colocalize on $\Delta i c m T$-containing vacuoles in neither Ax3 or $\Delta$ seyl cells (data not shown), confirming that these LCVs do not 
348 acquire ER (Weber et al., 2014b, Steiner et al., 2017). In summary, quantitative data from

349 IFC confirmed the confocal microscopy observations that Sey1 promotes the acquisition of 350 ER by LCVs harboring wild-type L. pneumophila.

351 Following the same procedure, we also assessed the time-dependent colocalization score of 352 P4C-mCherry on LCVs by IFC. The IFC colocalization score of P4C-mCherry on LCVs 353 formed by wild-type L. pneumophila increased during early time points of infection $(1 \mathrm{~h}-6 \mathrm{~h}$ 354 p.i.) (Figure $8 \mathrm{~b}$ ), while it decreased at later time points ( $24 \mathrm{~h}-48 \mathrm{~h}$ p.i.) (Figure S6e). There 355 was almost no difference in the P4C-mCherry colocalization score between infected $D$. 356 discoideum $\mathrm{Ax} 3$ and $\Delta$ sey1. Hence, Sey1 appears dispensable for the acquisition of 357 PtdIns(4)P to the LCV membrane. Finally, P4C-mCherry was not observed on vacuoles 358 formed by $\Delta i c m T$ mutant bacteria (data not shown), confirming that these LCVs do not 359 undergo PI conversion and are not decorated with PtdIns(4)P (Weber et al., 2014a, Steiner et 360 al., 2017). In summary, while the accumulation of calnexin-positive ER on LCVs in $D$. 361 discoideum is dependent on Sey1, the acquisition of $\operatorname{PtdIns}(4) P$ at the limiting LCV 362 membrane occurs independently of Sey1.

$364 \quad 2.10 \mathrm{LCV}$ expansion is delayed in D. discoideum $\Delta$ sey 1 cells

365 A hallmark of LCV maturation is its expansion to accommodate a growing number of 366 vacuolar bacteria. Comparing the LCV expansion in D. discoideum Ax3 and $\Delta s e y 1$, we found 367 that while in the parental strain the PtdIns(4)P-positive limiting LCV membrane expanded, in $368 \Delta$ seyl cells the PtdIns(4)P-positive membrane remained rather tightly wrapped around the 369 bacteria at early time points ( $2 \mathrm{~h}-16 \mathrm{~h}$ p.i.) and only later expanded ( $24 \mathrm{~h}-48 \mathrm{~h}$ p.i.) (Figures 370 8c, S6c, and S6d). These results indicate that Sey1 indeed promotes the expansion of the 371 PtdIns(4)P-positive LCV membrane early during infection but appears dispensable at later 372 stages of infection. The quantification of the LCV area revealed that at $2 \mathrm{~h}-16 \mathrm{~h}$ p.i., LCVs 
373 in $\Delta$ seyl cells were approximately 1.5 -fold smaller than in the parental strain (Figure $8 \mathrm{~d}$ ). At 374 later time points ( $24 \mathrm{~h}-48 \mathrm{~h}$ p.i.), the expansion defect disappeared, suggesting that Sey1 375 deletion only delays early LCV expansion (Figures 8c, 8d, S6c, and S6d). Taken together, 376 infection of dually labeled D. discoideum producing the PtdIns(4)P/LCV probe P4C-mCherry 377 and the ER marker calnexin-GFP revealed that in D. discoideum lacking Sey1 LCV expansion 378 is delayed early during infection. Hence, Sey1 plays a crucial role for LCV maturation and 379 intracellular replication of L. pneumophila.

\section{DISCUSSION}

382

383

384 385 386

\subsection{D. discoideum $\Delta s e y 1$ amoebae show an abnormal ER architecture}

D. discoideum deleted for Seyl were shown here to display an abnormal ER network morphology, with disorganized clumps of ER tubules at the cell periphery, as reported for several organisms following ATL gene deletion (Rismanchi et al., 2008, Hu et al., 2009, Wang et al., 2016, Zhao et al., 2016). This morphological defect was only rescued by overexpression of Seyl with a functional GTPase domain, consistent with the wellestablished ATL GTPase-dependent fusion of tubules leading to the formation of three-way junctions within tubules to create the cortical ER mesh. Unexpectedly, some ER branching still occurred in the absence of Sey1, indicating that Sey1-independent fusion events might partially compensate the loss of Sey1, thus ensuring cell viability. An alternative fusion pathway mediated by the ER SNAREs Ufe1p, Sec22p/Sec20p, Use1p and the tethering Dsl1 complex has been described in S. cerevisiae (Rogers et al., 2014). Since D. discoideum orthologs of these SNARES still localized to the ER in the absence of Sey1, a similar SNARE-based fusion process might exist in this amoeba.

The stabilization of ER tubules is dependent on reticulon (Rtn) and Yop1p/DP1/REEP families of ER-shaping proteins that directly interact with ATLs (Hu et al., 2009, Park et al., 
398 2010). The ER-network formation requires a finely tuned balance between ATL-dependent 399 tubule fusion and Rtn-mediated tubule curvature (Wang et al., 2016). D. discoideum 400 expresses only one Rtn ortholog (Rtnlc), and inhibition of Rtnlc functions by its deletion or 401 overexpression had no obvious consequences on the shape of ER tubules (Figures S1b and 402 S2c). The absence of defects in $\Delta$ rtnlc cells is consistent with the normal ER network 403 morphology observed in Rtn1p and Rtn2p deleted yeast cells (Voeltz et al., 2006). Moreover, 404 the loss of RTN4a/b in mouse embryonic fibroblasts induces a reduction of ER tubulation 405 (Jozsef et al., 2014), and the overexpression of the reticulons results in tubule fragmentation 406 (Wang et al., 2016, Espadas et al., 2019). This discrepancy suggests that besides Rtnlc, other 407 ER-shaping proteins might stabilize tubules in D. discoideum. PSI-BLAST searches did not 408 reveal any D. discoideum orthologs of DP1/Yop1/REEP proteins, and therefore, $\Delta r t n l c$ cells 409 might advantageously allow the identification of new tubule curvature mechanisms or factors. 410

\subsection{D. discoideum $\Delta$ sey1 amoebae upregulate the UPR pathway}

412 In mammalian cells, deletion of ATLs has been shown to induce the UPR pathway to limit 413 ER stress due to the delay in ER exit of some cargo proteins (Niu et al., 2019). Confirming 414 this observation, we show here that Seyl deletion caused the overexpression of the ERAD 415 protein $\mathrm{CdcC}$, indicative of a constitutively active UPR pathway. This possibly implies that 416 the ER morphological defects in $\Delta s e y l$ cells might interfere with proper cargo protein sorting 417 or folding in the ER. Interestingly, we observed that $\Delta$ seyl cells were unable to cope with 418 prolonged ER stress induced by tunicamycin. Besides the ERAD pathway, autophagy and ER 419 phagy/reticulophagy have been shown to contribute to the cellular response to ER stress 420 (Smith et al., 2017, Song et al., 2018, Stolz et al., 2019, Wilkinson, 2019, Wilkinson, 2020). 421 Hence, the selection of ER subdomains containing damaged or excess material is mediated by 422 several specific ER-phagy receptors including atlastin (ATL3) (Chen et al., 2019). Further 
423 studies will be required to assess whether autophagy and ER-phagy processes are altered in $424 \quad \Delta s e y 1$ cells.

425

426

\subsection{D. discoideum $\Delta$ sey1 amoebae are defective for growth on bacterial lawns}

D. discoideum $\Delta$ seyl cells had a marked growth defect when fed on bacteria and, to a lesser 428 extent, on axenic liquid culture medium. Our results indicate that at least two cumulative 429 defects in proteolysis and cell motility might account for these defective growth rates. First, 430 we established that reduced proteolytic capacities in $\Delta s e y l$ cells were correlated with 431 impaired intracellular retention of lysosomal enzymes due to increased lysosomal exocytosis 432 rates. In $D$. discoideum, lysosomes undergo several maturation steps to form post-lysosomes 433 which eventually become competent to fuse with the plasma membrane to expel their content. 434 Lysosomal exocytosis defects have been described in several $D$. discoideum mutants affecting 435 lysosomal maturation before lysosome exocytosis (Charette et al., 2007, Charette et al., 2008, 436 Carnell et al., 2011). Since no noticeable lysosomal maturation defects were observed in 437 Aseyl cells, a hypothetical ER-dependent step in the exocytosis process might be altered in 438 the absence of Sey1. For instance, the ER might provide key factors owing its close proximity 439 to lysosomes at membrane contact sites (MCS) (Phillips et al., 2016). The morphological 440 defects of the peripheral ER network in $\Delta s e y l$ cells might interfere with the formation, the 441 location or even the function of these ER-lysosome MCS.

442 The growth defect on bacterial lawns might also be amplified by the reduced cell motility 443 observed in $\Delta$ seyl cells. The ER network has been shown to maintain connections with the 444 actin and microtubule cytoskeletons, both required for cell motility. Several ER proteins, such 445 as CLIMP63, P180, REEP1, Sec61b, and STIM1 physically interact with microtubules (MTs) 446 (Klopfenstein et al., 1998, Ogawa-Goto et al., 2007, Grigoriev et al., 2008, Park et al., 2010, 447 Zhu et al., 2018), whereas SYP73 recruits the actin cytoskeleton in Arabidopsis (Cao et al., 
448 2016). A number of studies indicate that the ER can assist the cytoskeleton during organelle 449 and cell motility. For instance, spastin, an ATPase with MT-severing activity regulating MT 450 dynamics, has been shown to bind atlastin and REEP1 (Evans et al., 2006, Sanderson et al., 451 2006, Lee et al., 2009, Park et al., 2010), and its depletion affects the motility of 452 Glioblastoma cells (Draberova et al., 2011). Furthermore, in Arabidopsis, deletion of the 453 atlastin-related protein RDH3 prevents ER streaming during cell expansion (Stefano et al., 454 2014). Accordingly, the altered ER morphology of $\Delta s e y l$ cells might hamper the proper 455 mobilization of ER tubules to physically assist the cytoskeleton during cell migration.

456 In the context of host-pathogen interactions, phagocytes infected with L. pneumophila 457 were impaired for cell and organelle motility (Rothmeier et al., 2013, Swart et al., 2020b). A 458 family of RCC1 repeat containing L. pneumophila effectors has been shown to activate the 459 small GTPase Ran, and consequently, stabilize MTs and promote cell and organelle motility. 460 It is unknown whether L. pneumophila has evolved strategies to interfere with ER-associated 461 motility functions.

462

\section{$463 \quad 3.4$ D. discoideum $\Delta$ sey1 amoebae restrict growth of $\boldsymbol{L}$. pneumophila}

464 Inside eukaryotic host cells, the facultative intracellular bacterium L. pneumophila establishes 465 an ER-associated replication-permissive vacuole, the LCV. To this end, the pathogen employs 466 a multi-step process, including ATL3/Sey1-dependent ER recruitment (Steiner et al., 2017). 467 Given the various defects of $D$. discoideum $\Delta$ seyl cells in ER morphology and dynamics, we 468 assessed intracellular replication and LCV formation in the mutant amoebae. Indeed, $\Delta$ seyl 469 cells restricted the intracellular growth of L. pneumophila but not the killing of an avirulent 470 mutant strain (Figures $7 \mathrm{a}$ and $7 \mathrm{~b}$ ). TEM and confocal microscopy analyses as well as IFC 471 revealed a disrupted ER morphology in $\Delta s e y l$ cells and showed defective recruitment of ER 472 to LCVs, which was successfully complemented by plasmid-borne production of GFP-Sey1 
473 (Figures 7c, 8a, and 8b). Moreover, confocal microscopy analysis using dually labeled $D$. 474 discoideum producing in parallel the PtdIns(4)P/LCV probe P4C-mCherry and the ER marker 475 calnexin-GFP revealed that the expansion of LCVs formed in $\Delta$ seyl cells was significantly 476 delayed (Figures 8c and 8d).

477 These findings are in agreement with previous observations using D. discoideum 478 producing a catalytically-inactive, dominant negative variant of Sey1 (Sey1_K154A), which 479 was found to inhibit intracellular replication of L. pneumophila, as well as the decoration with 480 ER, the expansion and the aggregation/fusion of LCVs (Steiner et al., 2017, Steiner et al., 481 2018a). In addition, the results reported in this study rule out off-target effects of 482 Sey1_K154A, such as the formation of heterodimers with large GTPases other than Sey1. 483 Indeed, the depletion by RNA interference of another large GTPase, the mitochondrial fission 484 factor DNM1L (dynamin 1-like protein), has recently been shown to impair intracellular 485 replication of L. pneumophila (Escoll et al., 2017).

486 The mechanism underlying the role of Sey1 for LCV expansion is unknown. Sey1487 catalyzed ER dynamics might promote the budding, trafficking and/or fusion of ER-derived 488 vesicles, which interact with the pathogen vacuole (Kagan et al., 2002), and thus, contribute 489 to LCV maturation and expansion. Alternatively, the Sey1-catalyzed spatial vicinity of ER to 490 the PtdIns(4)P-positive pathogen vacuole might foster the formation of MCSs, which promote 491 lipid exchange and LCV expansion. Furthermore, our detailed analysis of the LCV maturation 492 kinetics by confocal microscopy indicated that the expansion of LCVs in the $\Delta$ seyl cells was 493 delayed only up to $16 \mathrm{~h}$ p.i. (Figures $8 \mathrm{c}$ and $8 \mathrm{~d}$ ). This defect appears to contribute to an 494 overall inhibition of intracellular replication of L. pneumophila in D. discoideum lacking Sey1 495 (Figure 7a). The replication defect appeared to be more pronounced in $\Delta$ seyl amoebae $(\sim 3-$ 496 fold decrease in replication) in comparison to the previously published defect caused by the 497 overproduction of the dominant negative Sey1 variant, Sey1_K154A ( 2 -fold decrease in 
498 replication) (Steiner et al., 2017). The reasons for a role of Sey1 in LCV expansion only at 499 rather early time points of infection and LCV maturation are unclear (Figures 8c, 8d, and S6). 500 Perhaps, fully functional ER architecture and dynamics are required only in the initial phase 501 to provide structural elements for LCV formation and expansion, nutrients for intracellular 502 growth, and/or protection from innate, host cell-autonomous defenses. Likely, the initial 503 phase of infection with a pathogen determines the outcome of the pathogen-host relationship, 504 i.e., that either the pathogen succeeds to establish a protective, replication-permissive niche, 505 or the host cell manages to kill the intruder.

506 In conclusion, in the present study we have characterized a number of phenotypes of $D$. 507 discoideum lacking Seyl. We showed that $\Delta$ seyl mutant amoebae are viable, exhibit 508 defective ER morphology and dynamics, reduced proteolytic processes and cell migration, as 509 well as impaired ER recruitment to LCVs, delay in pathogen vacuole expansion and reduced 510 intracellular replication of L. pneumophila. The successful generation of D. discoideum $\Delta$ sey 1 511 mutant cells paves the way for further detailed characterization of the role of Seyl in a variety 512 of cellular processes as well as for pathogen vacuole formation and maturation of different, 513 intracellular pathogens.

\section{EXPERIMENTAL PROCEDURES}

\section{$516 \quad 4.1$ Cell culture and $L$. pneumophila infections}

517 Detailed information on cell lines and bacterial strains used is reported in the Table S1. D. 518 discoideum strain Ax3 was grown at $22^{\circ} \mathrm{C}$ in $\mathrm{HL} 5$ medium or on lawns of live or heat-killed 519 bacteria as previously reported (Alibaud et al., 2008). For infections with L. pneumophila, D. 520 discoideum Ax3 and $\Delta$ seyl mutant amoebae were cultivated in HL5 medium (ForMedium) at $52123^{\circ} \mathrm{C}$ and transformed as previously described (Weber et al., 2014a, Weber et al., 2014b). 522 Transformation of two constructs in parallel was performed to obtain dually-fluorescent 
523 strains. Geneticin (G418, $20 \mu \mathrm{g} / \mathrm{mL})$ and hygromycin $(50 \mu \mathrm{g} / \mathrm{mL})$ was supplemented when 524 necessary.

525 L. pneumophila strains were grown for 3 days at $37^{\circ} \mathrm{C}$ on agar plates containing charcoal 526 yeast extract (CYE), buffered with $N$-(2-acetamido)-2-aminoethane sulfonic acid (ACES). For 527 infections, bacterial overnight cultures were prepared in ACES yeast extract (AYE) medium 528 at a starting $\mathrm{OD}_{600}$ of 0.1 and were grown $\left(21 \mathrm{~h}, 37^{\circ} \mathrm{C}\right)$ until early stationary phase $\left(2 \times 10^{9}\right.$ 529 bacteria/mL). Chloramphenicol (Cam; $5 \mu \mathrm{g} / \mathrm{mL})$ was supplemented when necessary.

530

\subsection{Cell surface and cell size analysis}

532 All cell lines were cultivated at low density a few days before analysis $\left(<10^{6}\right.$ cells $\left./ \mathrm{mL}\right)$. To 533 determine the surface of contact area between cells and culture plastic dishes, phase-contrast 534 images were analyzed using ImageJ. To measure cell size based on electric current exclusion 535 (CASY technology), cells were resuspended at a density of $5 \times 10^{5}$ cells $/ \mathrm{mL}$ in culture 536 medium. After $2 \mathrm{~h}$ shaking, $200 \mu \mathrm{L}$ cells were added to $10 \mathrm{~mL}$ CASYton solution and 537 analyzed using a CASY TTC instrument equipped with a $60 \mu \mathrm{m}$ diameter capillary (OMNI 538 Life Science, Germany), kindly provided by Nicolas Talarek, IGMM/CNRS Montpellier.

\subsection{Plasmid construction}

541 All plasmids are described in Table S1. Rtnlc, Sec22b, Use1, Ufe1 coding sequences were 542 obtained by PCR from a cDNA library with the corresponding oligonucleotides (Table S2), 543 cloned in the indicated vectors and sequenced. Plasmids kindly provided by colleagues 544 include pCsA-SibA (P. Cosson; Centre Médical Universitaire, Geneva, Switzerland), as well 545 as RFP-2 $\times$ FYVE and GFP-2×FYVE (J. King; University of Sheffield, Sheffield, UK). 546 Translational fusions of mRFPmars with wild-type Sey1 or the catalytically inactive mutant 547 (Sey1_K154A) were constructed by digestion of pBS001 or pNP108, respectively, with BglII 
548 and SpeI. Subsequently, the ORFs of Sey1 or Sey1_K154A were cloned into the BgIII and 549 SpeI sites of pDM318, yielding pBS003 and pBS004, respectively.

\subsection{Inactivation of sey 1 and rtnlc}

552 A schematic representation of the strategies followed for genetic inactivation of seyl and 553 rtnlc, and PCR based identification is given in Figure S7. sseyl cells (seyl gene ID: 554 DDB_G0279823) were generated as follows. To construct the knockout vector (pFL1439), 555 the 5 ' fragment was amplified from genomic DNA with sense (OL1135) and antisense 556 (OL1136) oligonucleotides (from position 1 to 595 of the genomic DNA) and cloned into 557 pBlueScript vector (Stratagene, La Jolla, CA). The 3' fragment was obtained by PCR using 558 the GFP-Sey1 plasmid as template with sense (OL1137) and antisense (OL1138) 559 oligonucleotides (from position 2005 to 2862 of the genomic DNA) and cloned into 560 pBlueScript containing the 5' fragment. After sequencing, the knockout vector was completed 561 by inserting the blasticidin resistance cassette between the two 5'and 3' fragments. The 562 resulting plasmid was linearized (KpnI and NotI digestion) and electroporated into Ax3 cells 563 as reported (Alibaud et al., 2003). Transformants were selected in presence of $10 \mu \mathrm{g} / \mathrm{mL}$ 564 blasticidin. Individual colonies were tested by PCR to confirm seyl gene replacement.

$565 \Delta r t n l c$ cells (rtnlc gene ID: DDB_G0293088) were generated as follows. To obtain the

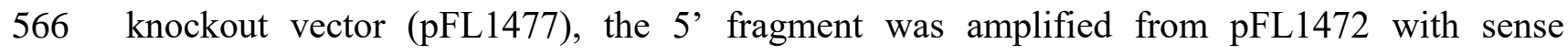
567 (OL1198) and antisense (OL1199) oligonucleotides (from position 192 to 584 of the genomic 568 DNA) and cloned into pBlueScript vector. The 3' fragment was obtained by PCR from 569 genomic DNA using sense (OL1200) and antisense (OL1201) oligonucleotides (from position 570852 to 1243 of the genomic DNA) and cloned into pBlueScript containing the 5' fragment. 571 After sequencing, the knockout vector was completed by inserting the blasticidin resistance 572 cassette between the two 5' and 3' fragments. Ax3 cells were electroporated with the 
573 linearized vector (KpnI and NotI digestion), and $\Delta r t n l c$ clones were selected as described 574 above for Sey1.

575

576

\subsection{Endocytosis, exocytosis, glycosidase and proteolytic activities}

577 Analysis of fluid phase (Alexa-Fluor-647-dextran; Molecular Probes) uptake/exocytosis, 578 phagocytosis of particles (YG-fluorescent 1- $\mu$ m latex beads; Polysciences) or GFP-producing 579 K. pneumoniae bacteria, was performed by flow cytometry as previously described (Lima et 580 al., 2012) (BD FACSCanto II, bdbiosciences). All data were normalized to protein content. 581 Intracellular and secreted glycosidase activities were measured as previously described 582 (Froquet et al., 2008, Le Coadic et al., 2013). CtsD activity was analyzed by measuring the 583 fluorescence release of a specific fluorogenic substrate (Mca-Gly-Lys-Pro-Ile-Leu-Phe-Phe584 Arg-Leu-Lys(Dnp)-D-Arg-NH2 [Mca = (7-methoxycoumarin-4-yl)acetyl; Dnp = dinitro585 phenyl]; BML-P145-0001, Enzo) with a microplate reader (Spark 20M, Tecan Life Sciences). 586 Lysozymes enzymatic activity in cell lysates was measured by the reduction of turbidity of 587 heat-killed Micrococcus lysodeikticus (Sigma) in suspension or on agarose plates as described 588 (Le Coadic et al., 2013, Bodinier et al., 2020). To measure intracellular proteolysis, $50 \mathrm{mg}$ 589 carboxylate-modified microspheres, $2.0 \mu \mathrm{m}$, blue fluorescent $(365 / 415 \mathrm{~nm})$ (FluoSpheres, 590 Molecular Probes, Thermofisher) were incubated overnight with $1 \mathrm{mg}$ DQ-Green-labeled 591 BSA (Invitrogen, Thermofisher) under continuous rotation. Coated beads were washed by 592 five cycles of low speed centrifugation and resuspension in culture medium. Cells were next 593 incubated with beads for $30 \mathrm{~min}$, washed, and further incubated for $30 \mathrm{~min}$. Intracellular 594 proteolysis of BSA released DQ-Green fluorescence $(505 / 515 \mathrm{~nm})$, and intrinsic fluorescence 595 of the beads $(365 / 415 \mathrm{~nm})$ were measured by flow cytometry.

596

$597 \quad 4.6$ Analysis of the UPR pathway 
598 To analyze cell viability upon tunicamycin $(\mathrm{TN})$ treatment, cells were seeded at $1-2 \times 10^{5}$ 599 cells/well on 24 wells tissue culture plates and treated with TN. At the indicated times, cells 600 were resuspended by pipetting, and propidium iodide was added for $10 \mathrm{~min}$ before flow 601 cytometry analysis.

602 Analysis of PDI, CdcD and EHD production was performed by Western blotting as 603 reported (Dominguez-Martin et al., 2018b). Briefly, cells were first seeded at $10^{6}$ cells/well 604 on 6-wells tissue culture plates. After incubation for $24 \mathrm{~h}$ with $1.5 \mu \mathrm{g} / \mathrm{mL} \mathrm{TN}$, cells were 605 washed once in phosphate buffer, and lysed for $30 \mathrm{~min}$ on ice in RIPA lysis buffer (10 $\mathrm{mM}$ 606 Tris- $\mathrm{HCl}, \mathrm{pH} 7.5,150 \mathrm{mM} \mathrm{NaCl}, 0.5 \mathrm{mM}$ EDTA, $0.5 \%$ NP-40, $0.05 \%$ SDS, protease 607 inhibitors) $(100 \mu \mathrm{L} /$ well). Protein concentrations were determined and adjusted before SDS 608 polyacrylamide electrophoresis and immunoblotting were performed as previously described 609 (Cornillon et al., 2000). Proteins were revealed by ECL (Thermo Scientific, Courtaboeuf, 610 France) and a ChemiDoc MP imager (Bio-Rad, Marnes-la-Coquette, France) allowing signal 611 quantification (ImageQuant software).

\subsection{Immunofluorescence microscopy}

614 Antibodies used in this study are described in Table S3. Anti-p97 (CdcD) and Alexa-Fluor615 647-coupled anti-p80 antibodies were kindly provided by L. Eichinger (University of 616 Cologne, Cologne, Germany) and P. Cosson (Centre Médical Universitaire, Geneva, 617 Switzerland), respectively. Actin was stained with TRITC-labeled phalloidin (Sigma618 Aldrich). For immunofluorescence analysis of uninfected D. discoideum, cells were fixed and 619 processed as previously detailed (Dias et al., 2013). Cells were observed with an inverted 620 Zeiss Axio Observer microscope or by confocal microscopy (either Leica SPE or Zeiss 621 Confocal ZEISS 880 with Fast Airyscan). 
623

624

625

626

627

628

629

630

631

632

633

634

635

636

637

638

639

640

641

642

644 cells were lysed with $0.8 \%$ saponin (Sigma-Aldrich) for $10 \mathrm{~min}$ at RT, and dilutions were

645 plated on CYE agar plates containing Cam $(5 \mu \mathrm{g} / \mathrm{mL})$ and incubated at $37^{\circ} \mathrm{C}$ for 3 days. CFUs

646 were assessed using an automated colony counter (CounterMat Flash 4000, IUL Instruments,

647 CounterMat software), and the number of CFUs (per $\mathrm{mL}$ ) was calculated. Using the same

\subsection{Live cell imaging and analysis}

To analyze cell motility, cells were deposed on $35 \mathrm{~mm}$ glass bottom dish (Ibidi) in filtered HL5 medium and imaged with a spinning disc Nikon Ti Andor CSU-W1 confocal microscope every $30 \mathrm{~s}$ for $30 \mathrm{~min}$. Films were processed by manual centroid tracking with the ImageJ plugin MTrackJ. To analyze microtubule and calnexin dynamics, cells were attached to glass coverslips in filtered HL5 medium for at least $30 \mathrm{~min}$ and overlaid with a $1 \mathrm{~mm}$ thin agarose sheet before observation. Images were taken every second for 2 min with a confocal ZEISS 880 microscope equipped with Fast Airyscan detection.

\subsection{Intracellular growth and killing of L. pneumophila in D. discoideum}

Intracellular replication of L. pneumophila JR32 and $\Delta i c m T$ in D. discoideum Ax3 and $\Delta$ seyl mutant cells was analyzed by colony forming units (CFUs) as well as by increase in relative fluorescent units (RFUs) following intravacuolar replication of mCherry-producing $L$. pneumophila strains. To determine CFUs, GFP-producing D. discoideum Ax3 or $\Delta$ seyl amoebae were seeded at a density of $1 \times 10^{5}$ cells $/ \mathrm{mL}$ in cell culture-treated 96-wells plates (VWR) and cultivated at $23^{\circ} \mathrm{C}$ in $\mathrm{HL} 5$ medium containing geneticin (G418, $\left.20 \mu \mathrm{g} / \mathrm{mL}\right)$. Afterwards, the amoebae were infected (MOI 1) with early stationary phase L. pneumophila JR32 or $\Delta i c m T$ constitutively producing mCherry, diluted in MB medium (Solomon et al., 2000), centrifuged (450 $\mathrm{g}, 10 \mathrm{~min}, \mathrm{RT}$ ) and incubated for $1 \mathrm{~h}$ at $25^{\circ} \mathrm{C}$. Subsequently, the infected amoebae were washed 3 times with $\mathrm{MB}$ medium and incubated at $25^{\circ} \mathrm{C}$ for the time indicated (96-wells plate was kept moist by addition of $\mathrm{ddH}_{2} \mathrm{O}$ in surrounding wells). The 
648 procedure, killing of mCherry-producing L. pneumophila $\Delta i c m T$ (MOI 50) was assessed in 649 GFP-producing D. discoideum Ax3 or $\Delta$ seyl. Following CFU counting, the percentage of 650 surviving bacteria (in relation to the initial bacteria number) was calculated for each time 651 point.

652 To determine increase in fluorescence derived from intracellular replication of mCherry653 producing L. pneumophila, GFP-producing D. discoideum Ax3 or $\Delta$ seyl amoebae were 654 seeded at a density of $1 \times 10^{5}$ cells $/ \mathrm{mL}$ in cell culture-treated 96-wells plates (VWR) and 655 cultivated at $23^{\circ} \mathrm{C}$ in HL5 medium containing geneticin (G418, $\left.20 \mu \mathrm{g} / \mathrm{mL}\right)$. The cells were 656 infected (MOI 1) with early stationary phase mCherry-producing L. pneumophila JR32, 657 diluted in MB medium, centrifuged $(450 \mathrm{~g}, 10 \mathrm{~min}, \mathrm{RT})$ and incubated for $1 \mathrm{~h}$ at $25^{\circ} \mathrm{C}$. 658 Afterwards, the infected amoebae were washed 3 times with fresh MB medium and incubated 659 at $25^{\circ} \mathrm{C}$ for the time indicated (96-wells plate was kept moist by addition of $\mathrm{ddH}_{2} \mathrm{O}$ in 660 surrounding wells). Increase in mCherry fluorescence was assessed every 2 days using a 661 microtiter plate reader (Synergy H1, Biotek).

662

6634.10 Confocal microscopy of infected cells

664 Infected D. discoideum amoebae were fixed and imaged by confocal microscopy. In short, 665 exponential phase $D$. discoideum Ax3 and $\Delta$ seyl amoebae producing calnexin-GFP and P4C666 mCherry were seeded in cell culture-treated 6-wells plates (VWR) at a density of $1 \times 10^{5}$ 667 cells $/ \mathrm{mL}$ or $2 \times 10^{5}$ cells $/ \mathrm{mL}$ respectively, and cultured overnight at $23^{\circ} \mathrm{C}$ in HL5 medium 668 containing geneticin $(\mathrm{G} 418,20 \mu \mathrm{g} / \mathrm{mL})$ and hygromycin $(50 \mu \mathrm{g} / \mathrm{mL})$. The cells were infected 669 (MOI 5) with mCerulean-producing L. pneumophila JR32 diluted in HL5, synchronized by 670 centrifugation $(450 \mathrm{~g}, 10 \mathrm{~min}, \mathrm{RT})$ and incubated at $25^{\circ} \mathrm{C}$ for $1 \mathrm{~h}$. Infected amoebae were then 671 washed 3 times with fresh HL5 and incubated at $25^{\circ} \mathrm{C}$ for the time indicated. Subsequently, 672 infected cells (including supernatant) were collected from the 6-wells plate, centrifuged (500 
$673 \mathrm{~g}, 5 \mathrm{~min}, \mathrm{RT}$ ) and fixed with 4\% PFA (Electron Microscopy Sciences) for $30 \mathrm{~min}$ at RT.

674 Fixed cells were then washed twice with Soerensen phosphate buffer (SorC) (Malchow et al., 675 1972), transferred to a 8-wells $\mu$-slide dish (Ibidi) and embedded under a layer of PBS/0.5\% 676 agarose.

677 The samples were imaged with a Leica TCS SP8 X CLSM (HC PL APO CS2, objective $67863 \times / 1.4-0.60$ oil; Leica Microsystems) with a scanning speed of $400 \mathrm{~Hz}$, bi-directional laser 679 scan and line accumulation equal 2. Pictures were acquired with a pinhole of 1.19 Airy Units 680 (AU) and with a pixel/voxel size close to the instrument's Nyquist criterion of $43 \times 43 \times 130 \mathrm{~nm}$ 681 (xyz). Images were deconvolved with Huygens professional version 19.10 software 682 (Scientific Volume Imaging, http://svi.nl) using the CMLE algorithm, set to 40 iterations and 683 quality threshold of 0.05. Z-stacks were finalized/exported using Imaris 9.5.0 software 684 (Bitplane).

685

$686 \quad 4.11$ Imaging flow cytometry

687 Processing of infected amoebae for imaging flow cytometry (IFC) was performed essentially 688 as described (Welin et al., 2018). In short, D. discoideum Ax3 and $\Delta$ sey1 mutant producing 689 calnexin-GFP and P4C-mCherry were seeded in T-75 flasks containing HL5 medium, 690 geneticin $(\mathrm{G} 418,20 \mu \mathrm{g} / \mathrm{mL})$ and hygromycin $(50 \mu \mathrm{g} / \mathrm{mL})$, and infected (MOI 5) with mPlum691 producing L. pneumophila JR32 diluted in HL5 medium. The infection was synchronized by 692 centrifugation ( $450 \mathrm{~g}, 10 \mathrm{~min}, \mathrm{RT})$, and the cells were incubated for $1 \mathrm{~h}$ at $25^{\circ} \mathrm{C}$. Infected cells 693 were then washed three times with HL5 and incubated at $25^{\circ} \mathrm{C}$ for the time indicated. 694 Afterwards, cells were collected, centrifuged (500 g, 5 min, RT) and fixed in 2\% PFA for 90 695 min on ice. Fixed infected amoebae were washed twice in phosphate-buffered saline (PBS) 696 and resuspended in $20 \mu \mathrm{L}$ ice-cold PBS prior to IFC analysis. 
697 Using an imaging flow cytometer (ImageStreamX MkII; Amnis), at least 5'000 cells were 698 acquired and analyzed with the IDEAS (v.6.2) software (Amnis). In short, infected amoebae 699 containing one intracellular L. pneumophila bacterium were gated and analyzed for 700 colocalization of GFP and mCherry (host) with mPlum produced by L. pneumophila 701 (minimum number of gated events specified in the figure legend). The software computes the 702 IFC colocalization score (bright detail similarity), which is the log-transformed Pearson's 703 correlation coefficient of the localized bright spots with a radius of 3 pixels or less in two 704 images, and is used to quantify relative enrichment of a marker on the LCV. Data analysis 705 was performed using GraphPad Prism. Regular two-way ANOVA followed by Bonferroni 706 post-hoc test was used to compare all samples between each other.

707

$708 \quad 4.12$ Electron microscopy

709 L. pneumophila-infected D. discoideum Ax3, $\Delta$ seyl producing GFP or $\Delta$ seyl producing GFP710 Sey1 were fixed with $4 \%$ PFA and $0.1 \%$ glutaraldehyde in $0.1 \mathrm{M}$ sodium cacodylate buffer $711(\mathrm{pH} 7.35)$ for $30 \mathrm{~min}$ at $4^{\circ} \mathrm{C}$. Afterwards, the samples were treated sequentially with $2.5 \%$ 712 glutaraldehyde for $30 \mathrm{~min}, 1 \% \mathrm{OsO}_{4}$ for $1 \mathrm{~h}$ at $0^{\circ} \mathrm{C}$, both in $0.1 \mathrm{M}$ sodium cacodylate buffer 713 and $2 \%$ uranyl acetate in $\mathrm{ddH}_{2} \mathrm{O}$ for $1 \mathrm{~h}$ at $4{ }^{\circ} \mathrm{C}$, followed by embedment in $2 \%$ Difco Noble 714 agar (BD Biosciences, Allschwil, Switzerland) in $\mathrm{H}_{2} \mathrm{O}$ to form an immobilized pellet. An 715 increasing ethanol series (50\%-100\%) was then used to dehydrate the samples, which were 716 finally embedded in Epon/Araldite (Sigma-Aldrich). Contrast enhancement of ultrathin (50 $717 \mathrm{~nm}$ ) sections was done using Reynolds lead citrate. Processed samples were examined with a 718 CM100 transmission electron microscope (Thermo Fisher Scientific, Eindhoven, The 719 Netherlands) at an acceleration voltage of $80 \mathrm{kV}$ using an Orius 1000 digital camera (Ametek $720 \mathrm{GmbH}$, Munich, Germany). 


\section{ACKNOWLEDGEMENTS}

723 Confocal laser scanning microscopy and TEM was performed using equipment of the Center 724 of Microscopy and Image Analysis, University of Zurich (UZH), run by Andres Kaech and 725 Urs Ziegler. Imaging Flow Cytometry was performed at the Flow Cytometry Core Facility of 726 the Faculty of Medicine and Health Sciences, Linköping University, Linköping, Sweden. 727 Flow cytometry and microscopy analyses of uninfected cells were performed at the 728 Montpellier RIO imaging facility of the University of Montpellier, member of the national 729 infrastructure France-BioImaging, supported by the French National Research Agency (ANR730 10-INBS-04, "Investments for the future"). Work in the group of H.H. was supported by the 731 Swiss National Science Foundation (SNF; 31003A_175557), the Novartis Foundation for 732 Medical-Biological Research, and the OPO foundation. Work in the group of V.M. and F.L. 733 was supported by the Région Occitanie. A.W. was supported by grants from the Swedish 734 Society of Medicine (SLS-934829) and the Ake Wiberg Foundation. The funders had no role 735 in study design, data collection and analysis, decision to publish, or preparation of the 736 manuscript.

738 AUTHORS' CONTRIBUTION

739 H.H. and F.L. conceived the study and guided experiments. D.H., Be.S., A.W., Bi.S., A.L.S. 740 and F.L. designed and conducted experiments. V.M. provided funding. H.H. and F.L. wrote 741 the paper with input from all other authors.

742

743 CONFLIT OF INTEREST

744 The authors declare no conflict of interest. 


\section{REFERENCES}

747 Al-Quadan, T., Price, C.T. and Abu Kwaik, Y. (2012). Exploitation of evolutionarily 748 conserved amoeba and mammalian processes by Legionella. Trends in Microbiology 20, $749 \quad 299-306$.

750 Alibaud, L., Cosson, P. and Benghezal, M. (2003). Dictyostelium discoideum transformation 751 by oscillating electric field electroporation. BioTechniques 35, 78-80, 82-73.

752 Alibaud, L., Kohler, T., Coudray, A., Prigent-Combaret, C., Bergeret, E., Perrin, J., et al. 753 (2008). Pseudomonas aeruginosa virulence genes identified in a Dictyostelium host model. $754 \quad$ Cellular Microbiology 10, 729-740.

755 Almanza, A., Carlesso, A., Chintha, C., Creedican, S., Doultsinos, D., Leuzzi, B., et al. 756 (2019). Endoplasmic reticulum stress signalling - from basic mechanisms to clinical 757 applications. The FEBS Journal 286, 241-278.

758 Anwar, K., Klemm, R.W., Condon, A., Severin, K.N., Zhang, M., Ghirlando, R., et al. (2012). 759 The dynamin-like GTPase Sey1p mediates homotypic ER fusion in S. cerevisiae. The $760 \quad$ Journal of Cell Biology 197, 209-217.

761 Asrat, S., de Jesus, D.A., Hempstead, A.D., Ramabhadran, V. and Isberg, R.R. (2014). 762 Bacterial pathogen manipulation of host membrane trafficking. Annual Review of Cell and 763 Developmental Biology 30, 79-109.

764 Bärlocher, K., Welin, A. and Hilbi, H. (2017). Formation of the Legionella replicative 765 compartment at the crossroads of retrograde trafficking. Frontiers in Cellular and Infection $766 \quad$ Microbiology 7, 482.

767 Bian, X., Klemm, R.W., Liu, T.Y., Zhang, M., Sun, S., Sui, X., et al. (2011). Structures of the 768 atlastin GTPase provide insight into homotypic fusion of endoplasmic reticulum 769 membranes. Proceedings of the National Academy of Sciences of the United States of $770 \quad$ America 108, 3976-3981. 
771 Bodinier, R., Leiba, J., Sabra, A., Jauslin, T.N., Lamrabet, O., Guilhen, C., et al. (2020). 772 LrrkA, a kinase with leucine-rich repeats, links folate sensing with Kil2 activity and 773 intracellular killing. Cellular Microbiology 22, e13129.

774 Byrnes, L.J., Singh, A., Szeto, K., Benvin, N.M., O'Donnell, J.P., Zipfel, W.R. and 775 Sondermann, H. (2013). Structural basis for conformational switching and GTP loading of 776 the large $\mathrm{G}$ protein atlastin. The EMBO Journal 32, 369-384.

777 Byrnes, L.J. and Sondermann, H. (2011). Structural basis for the nucleotide-dependent 778 dimerization of the large G protein atlastin-1/SPG3A. Proceedings of the National 779 Academy of Sciences of the United States of America 108, 2216-2221.

780 Cao, P., Renna, L., Stefano, G. and Brandizzi, F. (2016). SYP73 Anchors the ER to the actin 781 cytoskeleton for maintenance of ER integrity and streaming in Arabidopsis. Current 782 Biology 26, 3245-3254.

783 Cardenal-Munoz, E., Barisch, C., Lefrancois, L.H., Lopez-Jimenez, A.T. and Soldati, T. 784 (2017). When Dicty met Myco, a (not so) romantic story about one amoeba and its 785 intracellular pathogen. Frontiers in Cellular and Infection Microbiology 7, 529.

786 Carnell, M., Zech, T., Calaminus, S.D., Ura, S., Hagedorn, M., Johnston, S.A., et al. (2011). 787 Actin polymerization driven by WASH causes V-ATPase retrieval and vesicle 788 neutralization before exocytosis. The Journal of Cell Biology 193, 831-839.

789 Charette, S.J. and Cosson, P. (2007). A LYST/beige homolog is involved in biogenesis of 790 Dictyostelium secretory lysosomes. Journal of Cell Science 120, 2338-2343.

791 Charette, S.J. and Cosson, P. (2008). Altered composition and secretion of lysosome-derived 792 compartments in Dictyostelium AP-3 mutant cells. Traffic 9, 588-596.

793 Chen, J., Stefano, G., Brandizzi, F. and Zheng, H. (2011). Arabidopsis RHD3 mediates the 794 generation of the tubular ER network and is required for Golgi distribution and motility in 795 plant cells. Journal of Cell Science 124, 2241-2252. 
796 Chen, Q., Xiao, Y., Chai, P., Zheng, P., Teng, J. and Chen, J. (2019). ATL3 is a tubular ER797 phagy receptor for GABARAP-mediated selective autophagy. Current Biology 29, 846$798 \quad 855$.

799 Cornillon, S., Pech, E., Benghezal, M., Ravanel, K., Gaynor, E., Letourneur, F., et al. (2000). $800 \quad$ Phg1p is a nine-transmembrane protein superfamily member involved in Dictyostelium 801 adhesion and phagocytosis. The Journal of Biological Chemistry 275, 34287-34292.

802 Dias, M., Blanc, C., Thazar-Poulot, N., Ben Larbi, S., Cosson, P. and Letourneur, F. (2013). 803 Dictyostelium ACAP-A is an ArfGAP involved in cytokinesis, cell migration and actin 804 cytoskeleton dynamics. Journal of Cell Science 126, 756-766.

805 Dominguez-Martin, E., Hernandez-Elvira, M., Vincent, O., Coria, R. and Escalante, R. 806 (2018a). Unfolding the endoplasmic reticulum of a social amoeba: Dictyostelium 807 discoideum as a new model for the study of endoplasmic reticulum stress. Cells 7, 56.

808 Dominguez-Martin, E., Ongay-Larios, L., Kawasaki, L., Vincent, O., Coello, G., Coria, R. 809 and Escalante, R. (2018b). IreA controls endoplasmic reticulum stress-induced autophagy 810 and survival through homeostasis recovery. Molecular and Cellular Biology 38, e0005481118.

812 Draberova, E., Vinopal, S., Morfini, G., Liu, P.S., Sladkova, V., Sulimenko, T., et al. (2011). 813 Microtubule-severing ATPase spastin in glioblastoma: increased expression in human 814 glioblastoma cell lines and inverse roles in cell motility and proliferation. The Journal of $815 \quad$ Neuropathology and Experimental Neurology 70, 811-826.

816 English, A.R., Zurek, N. and Voeltz, G.K. (2009). Peripheral ER structure and function. 817 Current Opinion in Cell Biology 21, 596-602.

818 Escoll, P., Song, O.R., Viana, F., Steiner, B., Lagache, T., Olivo-Marin, J.C., et al. (2017). 819 Legionella pneumophila modulates mitochondrial dynamics to trigger metabolic 820 repurposing of infected macrophages. Cell Host \& Microbe 22, 302-316. 
821 Espadas, J., Pendin, D., Bocanegra, R., Escalada, A., Misticoni, G., Trevisan, T., et al. (2019).

822 Dynamic constriction and fission of endoplasmic reticulum membranes by reticulon. Nat $823 \quad$ Commun 10, 5327.

824 Evans, K., Keller, C., Pavur, K., Glasgow, K., Conn, B. and Lauring, B. (2006). Interaction of 825 two hereditary spastic paraplegia gene products, spastin and atlastin, suggests a common 826 pathway for axonal maintenance. Proceedings of the National Academy of Sciences of the 827 United States of America 103, 10666-10671.

828 Fink, J.K. (2013). Hereditary spastic paraplegia: clinico-pathologic features and emerging 829 molecular mechanisms. Acta Neuropathology 126, 307-328.

830 Finsel, I. and Hilbi, H. (2015). Formation of a pathogen vacuole according to Legionella 831 pneumophila: how to kill one bird with many stones. Cellular Microbiology 17, 935-950.

832 Fischer, D., Schabhuttl, M., Wieland, T., Windhager, R., Strom, T.M. and Auer-Grumbach, 833 M. (2014). A novel missense mutation confirms ATL3 as a gene for hereditary sensory $834 \quad$ neuropathy type 1. Brain 137, e286.

835 Froquet, R., Cherix, N., Birke, R., Benghezal, M., Cameroni, E., Letourneur, F., et al. (2008). 836 Control of cellular physiology by TM9 proteins in yeast and Dictyostelium. The Journal of 837 Biological Chemistry 283, 6764-6772.

838 Froquet, R., le Coadic, M., Perrin, J., Cherix, N., Cornillon, S. and Cosson, P. (2012). 839 TM9/Phg1 and SadA proteins control surface expression and stability of SibA adhesion 840 molecules in Dictyostelium. Molecular Biology of the Cell 23, 679-686.

841 Gomez-Valero, L., Rusniok, C., Cazalet, C. and Buchrieser, C. (2011). Comparative and 842 functional genomics of Legionella identified eukaryotic like proteins as key players in 843 host-pathogen interactions. Frontiers in Microbiology 2, 208. 
844 Grigoriev, I., Gouveia, S.M., van der Vaart, B., Demmers, J., Smyth, J.T., Honnappa, S., et al. 845 (2008). STIM1 is a MT-plus-end-tracking protein involved in remodeling of the ER. $846 \quad$ Current Biology 18, 177-182.

847 Guelly, C., Zhu, P.P., Leonardis, L., Papic, L., Zidar, J., Schabhuttl, M., et al. (2011). 848 Targeted high-throughput sequencing identifies mutations in atlastin-1 as a cause of 849 hereditary sensory neuropathy type I. American Journal of Human Genetics 88, 99-105.

850 Hoffmann, C., Finsel, I., Otto, A., Pfaffinger, G., Rothmeier, E., Hecker, M., et al. (2014). 851 Functional analysis of novel Rab GTPases identified in the proteome of purified 852 Legionella-containing vacuoles from macrophages. Cellular Microbiology 16, 1034-1052. 853 Hu, J., Shibata, Y., Zhu, P.P., Voss, C., Rismanchi, N., Prinz, W.A., et al. (2009). A class of 854 dynamin-like GTPases involved in the generation of the tubular ER network. Cell 138, $855 \quad 549-561$.

856 Isberg, R.R., O'Connor, T.J. and Heidtman, M. (2009). The Legionella pneumophila 857 replication vacuole: making a cosy niche inside host cells. Nature Reviews Microbiology 7, $858 \quad 13-24$.

859 Journet, A., Chapel, A., Jehan, S., Adessi, C., Freeze, H., Klein, G. and Garin, J. (1999). 860 Characterization of Dictyostelium discoideum cathepsin D. Journal of Cell Science 112, $861 \quad 3833-3843$.

862 Jozsef, L., Tashiro, K., Kuo, A., Park, E.J., Skoura, A., Albinsson, S., et al. (2014). Reticulon 8634 is necessary for endoplasmic reticulum tubulation, STIM1-Orai1 coupling, and store864 operated calcium entry. The Journal of Biological Chemistry 289, 9380-9395.

865 Kagan, J.C. and Roy, C.R. (2002). Legionella phagosomes intercept vesicular traffic from 866 endoplasmic reticulum exit sites. Nature Cell Biology 4, 945-954. 
867 Kagan, J.C., Stein, M.P., Pypaert, M. and Roy, C.R. (2004). Legionella subvert the functions 868 of Rab1 and Sec22b to create a replicative organelle. The Journal of Experimental $869 \quad$ Medicine 199, 1201-1211.

870 Kim, K.T., Moon, Y., Jang, Y., Lee, K.T., Lee, C., Jun, Y. and Lee, S. (2017). Molecular 871 mechanisms of atlastin-mediated ER membrane fusion revealed by a FRET-based single$872 \quad$ vesicle fusion assay. Scientific Reports 7, 8700.

873 Klemm, R.W., Norton, J.P., Cole, R.A., Li, C.S., Park, S.H., Crane, M.M., et al. (2013). A 874 conserved role for atlastin GTPases in regulating lipid droplet size. Cell Reports 3, 1465$875 \quad 1475$.

876 Klopfenstein, D.R., Kappeler, F. and Hauri, H.P. (1998). A novel direct interaction of 877 endoplasmic reticulum with microtubules. The EMBO Journal 17, 6168-6177.

878 Krols, M., Detry, S., Asselbergh, B., Almeida-Souza, L., Kremer, A., Lippens, S., et al. 879 (2018). Sensory-neuropathy-causing mutations in ATL3 cause aberrant ER membrane $880 \quad$ tethering. Cell Reports 23, 2026-2038.

881 Lai, Y.S., Stefano, G. and Brandizzi, F. (2014). ER stress signaling requires RHD3, a 882 functionally conserved ER-shaping GTPase. Journal of Cell Science 127, 3227-3232.

883 Lamrabet, O., Jauslin, T., Lima, W.C., Leippe, M. and Cosson, P. (2020). The multifarious 884 lysozyme arsenal of Dictyostelium discoideum. Developmental and Comparative $885 \quad$ Immunology 107, 103645.

886 Le Coadic, M., Froquet, R., Lima, W.C., Dias, M., Marchetti, A. and Cosson, P. (2013). 887 Phg1/TM9 proteins control intracellular killing of bacteria by determining cellular levels of 888 the Kil1 sulfotransferase in Dictyostelium. PloS One 8, e53259.

889 Lee, M., Paik, S.K., Lee, M.J., Kim, Y.J., Kim, S., Nahm, M., et al. (2009). Drosophila 890 atlastin regulates the stability of muscle microtubules and is required for synapse 891 development. Developmental Biology 330, 250-262. 
892 Liang, J.R., Lingeman, E., Ahmed, S. and Corn, J.E. (2018). Atlastins remodel the 893 endoplasmic reticulum for selective autophagy. The Journal of Cell Biology 217, 33548943367.

895 Lima, W.C., Leuba, F., Soldati, T. and Cosson, P. (2012). Mucolipin controls lysosome 896 exocytosis in Dictyostelium. Journal of Cell Science 125, 2315-2322.

897 Liu, T.Y., Bian, X., Romano, F.B., Shemesh, T., Rapoport, T.A. and Hu, J. (2015). Cis and 898 trans interactions between atlastin molecules during membrane fusion. Proceedings of the $899 \quad$ National Academy of Sciences of the United States of America 112, E1851-1860.

900 Liu, T.Y., Bian, X., Sun, S., Hu, X., Klemm, R.W., Prinz, W.A., et al. (2012). Lipid 901 interaction of the $\mathrm{C}$ terminus and association of the transmembrane segments facilitate 902 atlastin-mediated homotypic endoplasmic reticulum fusion. Proceedings of the National 903 Academy of Sciences of the United States of America 109, E2146-2154.

904 Malchow, D., Nagele, B., Schwarz, H. and Gerisch, G. (1972). Membrane-bound cyclic AMP 905 phosphodiesterase in chemotactically responding cells of Dictyostelium discoideum. 906 European Journal of Biochemistry / FEBS 28, 136-142.

907 Niu, L., Ma, T., Yang, F., Yan, B., Tang, X., Yin, H., et al. (2019). Atlastin-mediated 908 membrane tethering is critical for cargo mobility and exit from the endoplasmic reticulum. 909 Proceedings of the National Academy of Sciences of the United States of America 116, $910 \quad 14029-14038$.

911 O'Donnell, J.P., Byrnes, L.J., Cooley, R.B. and Sondermann, H. (2018). A hereditary spastic 912 paraplegia-associated atlastin variant exhibits defective allosteric coupling in the catalytic 913 core. The Journal of Biological Chemistry 293, 687-700.

914 Ogawa-Goto, K., Tanaka, K., Ueno, T., Tanaka, K., Kurata, T., Sata, T. and Irie, S. (2007). 915 p180 is involved in the interaction between the endoplasmic reticulum and microtubules 
916 through a novel microtubule-binding and bundling domain. Molecular Biology of the Cell $917 \quad \mathbf{1 8}, 3741-3751$.

918 Orso, G., Pendin, D., Liu, S., Tosetto, J., Moss, T.J., Faust, J.E., et al. (2009). Homotypic 919 fusion of ER membranes requires the dynamin-like GTPase atlastin. Nature 460, 978-983. 920 Park, S.H., Zhu, P.P., Parker, R.L. and Blackstone, C. (2010). Hereditary spastic paraplegia 921 proteins REEP1, spastin, and atlastin-1 coordinate microtubule interactions with the 922 tubular ER network. The Journal of Clinical Investigation 120, 1097-1110.

923 Pawar, S., Ungricht, R., Tiefenboeck, P., Leroux, J.C. and Kutay, U. (2017). Efficient protein 924 targeting to the inner nuclear membrane requires atlastin-dependent maintenance of ER 925 topology. eLife 6, e28202.

926 Personnic, N., Bärlocher, K., Finsel, I. and Hilbi, H. (2016). Subversion of retrograde 927 trafficking by translocated pathogen effectors. Trends in Microbiology 24, 450-462.

928 Phillips, M.J. and Voeltz, G.K. (2016). Structure and function of ER membrane contact sites 929 with other organelles. Nature Reviews Molecular and Cell Biology 17, 69-82.

930 Rismanchi, N., Soderblom, C., Stadler, J., Zhu, P.P. and Blackstone, C. (2008). Atlastin 931 GTPases are required for Golgi apparatus and ER morphogenesis. Human molecular 932 genetics 17, 1591-1604.

933 Robinson, C.G. and Roy, C.R. (2006). Attachment and fusion of endoplasmic reticulum with 934 vacuoles containing Legionella pneumophila. Cellular Microbiology 8, 793-805.

935 Rogers, J.V., McMahon, C., Baryshnikova, A., Hughson, F.M. and Rose, M.D. (2014). ER936 associated retrograde SNAREs and the Dsl1 complex mediate an alternative, Sey1p937 independent homotypic ER fusion pathway. Molecular Biology of the Cell 25, 3401-3412. 938 Rothmeier, E., Pfaffinger, G., Hoffmann, C., Harrison, C.F., Grabmayr, H., Repnik, U., et al. 939 (2013). Activation of Ran GTPase by a Legionella effector promotes microtubule 940 polymerization, pathogen vacuole motility and infection. PLoS Pathogens 9, e1003598. 
941 Salinas, S., Proukakis, C., Crosby, A. and Warner, T.T. (2008). Hereditary spastic paraplegia: 942 clinical features and pathogenetic mechanisms. Lancet Neurology 7, 1127-1138.

943 Sanderson, C.M., Connell, J.W., Edwards, T.L., Bright, N.A., Duley, S., Thompson, A., et al. 944 (2006). Spastin and atlastin, two proteins mutated in autosomal-dominant hereditary 945 spastic paraplegia, are binding partners. Human molecular genetics 15, 307-318.

946 Schwarz, D.S. and Blower, M.D. (2016). The endoplasmic reticulum: structure, function and 947 response to cellular signaling. Cellular and Molecular Life Sciences 73, 79-94.

948 Sherwood, R.K. and Roy, C.R. (2016). Autophagy evasion and endoplasmic reticulum 949 subversion: the Yin and Yang of Legionella intracellular infection. Annual Reviews in $950 \quad$ Microbiology 70, 413-433.

951 Shibata, Y., Hu, J., Kozlov, M.M. and Rapoport, T.A. (2009). Mechanisms shaping the 952 membranes of cellular organelles. Annual Reviews of Cell and Developmental Biology 25, $953 \quad 329-354$.

954 Shibata, Y., Voeltz, G.K. and Rapoport, T.A. (2006). Rough sheets and smooth tubules. Cell $955 \mathbf{1 2 6}, 435-439$.

956 Smith, M. and Wilkinson, S. (2017). ER homeostasis and autophagy. Essays in Biochemistry $957 \quad 61,625-635$.

958 Solomon, J.M. and Isberg, R.R. (2000). Growth of Legionella pneumophila in Dictyostelium 959 discoideum: a novel system for genetic analysis of host-pathogen interactions. Trends in $960 \quad$ Microbiology 8, 478-480.

961 Song, S., Tan, J., Miao, Y. and Zhang, Q. (2018). Crosstalk of ER stress-mediated autophagy 962 and ER-phagy: Involvement of UPR and the core autophagy machinery. Journal of Cell $963 \quad$ Physiology 233, 3867-3874.

964 Stefano, G., Renna, L. and Brandizzi, F. (2014). The endoplasmic reticulum exerts control 965 over organelle streaming during cell expansion. Journal of Cell Science 127, 947-953. 
966 Stefano, G., Renna, L., Lai, Y., Slabaugh, E., Mannino, N., Buono, R.A., et al. (2015). ER 967 network homeostasis is critical for plant endosome streaming and endocytosis. Cell 968 Discovery 1, 15033.

969 Steiner, B., Swart, A.L., Welin, A., Weber, S., Personnic, N., Kaech, A., et al. (2017). ER 970 remodeling by the large GTPase atlastin promotes vacuolar growth of Legionella 971 pneumophila. EMBO Reports 18, 1817-1836.

972 Steiner, B., Weber, S. and Hilbi, H. (2018a). Formation of the Legionella-containing vacuole: 973 phosphoinositide conversion, GTPase modulation and ER dynamics. International Journal 974 of Medical Microbiology 308, 49-57.

975 Steiner, B., Weber, S., Kaech, A., Ziegler, U. and Hilbi, H. (2018b). The large GTPase 976 atlastin controls ER remodeling around a pathogen vacuole. Communicative \& Integrative 977 Biology 11, 1-5.

978 Stolz, A. and Grumati, P. (2019). The various shades of ER-phagy. The FEBS Journal 286, $979 \quad 4642-4649$.

980 Swanson, M.S. and Isberg, R.R. (1995). Association of Legionella pneumophila with the 981 macrophage endoplasmic reticulum. Infection and Immunity 63, 3609-3620.

982 Swart, A.L., Harrison, C.F., Eichinger, L., Steinert, M. and Hilbi, H. (2018). Acanthamoeba 983 and Dictyostelium as cellular models for Legionella infection. Frontiers in Cellular and $984 \quad$ Infection Microbiology 8, 61.

985 Swart, A.L. and Hilbi, H. (2020a). Phosphoinositides and the fate of Legionella in 986 phagocytes. Frontiers in Immunology 11, 25.

987 Swart, A.L., Steiner, B., Gomez-Valero, L., Schutz, S., Hannemann, M., Janning, P., et al. 988 (2020b). Divergent evolution of Legionella RCC1 repeat effectors defines the range of Ran 989 GTPase cycle targets. mBio 11, e00405-20. 
990 Voeltz, G.K., Prinz, W.A., Shibata, Y., Rist, J.M. and Rapoport, T.A. (2006). A class of 991 membrane proteins shaping the tubular endoplasmic reticulum. Cell 124, 573-586.

992 Voss, C., Lahiri, S., Young, B.P., Loewen, C.J. and Prinz, W.A. (2012). ER-shaping proteins 993 facilitate lipid exchange between the ER and mitochondria in S. cerevisiae. Journal of Cell $994 \quad$ Science 125, 4791-4799.

995 Wang, S., Tukachinsky, H., Romano, F.B. and Rapoport, T.A. (2016). Cooperation of the ER996 shaping proteins atlastin, lunapark, and reticulons to generate a tubular membrane network. $997 \quad$ eLife 5, e18605.

998 Weber, S. and Hilbi, H. (2014a). Live cell imaging of phosphoinositide dynamics during 999 Legionella infection. Methods in Molecular Biology 1197, 153-167.

1000 Weber, S., Steiner, B., Welin, A. and Hilbi, H. (2018). Legionella-containing vacuoles 1001 capture PtdIns(4)P-rich vesicles derived from the Golgi apparatus. mBio 9, e02420-18.

1002 Weber, S., Wagner, M. and Hilbi, H. (2014b). Live-cell imaging of phosphoinositide 1003 dynamics and membrane architecture during Legionella infection. mBio 5, e00839-13.

1004 Welin, A., Weber, S. and Hilbi, H. (2018). Quantitative imaging flow cytometry of 1005 Legionella-infected Dictyostelium amoebae reveals the impact of retrograde trafficking on 1006 pathogen vacuole composition. Applied and Environmental Microbiology 84, e00158-18.

1007 Wilkinson, S. (2019). ER-phagy: shaping up and destressing the endoplasmic reticulum. The 1008 FEBS Journal 286, 2645-2663.

1009 Wilkinson, S. (2020). Emerging principles of selective ER autophagy. Journal of Molecular 1010 Biology 432, 185-205.

1011 Zhang, D., Vjestica, A. and Oliferenko, S. (2010). The cortical ER network limits the 1012 permissive zone for actomyosin ring assembly. Current Biology 20, 1029-1034.

1013 Zhang, M., Wu, F., Shi, J., Zhu, Y., Zhu, Z., Gong, Q. and Hu, J. (2013). ROOT HAIR 1014 DEFECTIVE3 family of dynamin-like GTPases mediates homotypic endoplasmic 
1015 reticulum fusion and is essential for Arabidopsis development. Plant Physiology 163, 7131016720.

1017 Zhao, G., Zhu, P.P., Renvoise, B., Maldonado-Baez, L., Park, S.H. and Blackstone, C. (2016). 1018 Mammalian knock out cells reveal prominent roles for atlastin GTPases in ER network 1019 morphology. Experimental Cell Research 349, 32-44.

1020 Zhu, Y., Zhang, G., Lin, S., Shi, J., Zhang, H. and Hu, J. (2018). Sec61beta facilitates the 1021 maintenance of endoplasmic reticulum homeostasis by associating microtubules. Protein 1022 \& Cell 9, 616-628.

1023

1024 


\section{FIGURE LEGENDS}

1026 FIGURE 1 Genomic deletion of D. discoideum seyl and basic mutant characterization.

1027 (a) Schematic representation of the strategy used to inactivate the $D$. discoideum seyl gene by 1028 insertion of the blasticidin resistance-encoding gene (BsR) by double recombination. Sey1 1029 comprises a large guanosine triphosphatase (GTPase) domain followed by a three-helix 1030 bundle (3HB) and two adjacent transmembrane motifs (TMs). In $\Delta$ seyl cells, the protein 1031 sequence from amino acid residues P149 to K588 is deleted. (b) Phase contrast microscopy 1032 and (c) histogram of cell-contact area of live cells on tissue culture dishes determined after 1033 binarization of images (ImageJ) $(\mathrm{n}=100)$ revealed that $\Delta$ seyl cells are enlarged compared to 1034 parental cells (Ax3). Scale bar, $10 \mu \mathrm{m}$. (d) Representative distribution of cell diameters 1035 measured by electric current exclusion (CASY technology). Compared to Ax3 cells, $\Delta$ sey 1 1036 cells show a greater diameter heterogeneity with a mean diameter of $10.24 \pm 0.15 \mu \mathrm{m}$ instead 1037 of $9.51 \pm 0.24 \mu \mathrm{m}$ in Ax3 cells (data represent the average and s.e.m. of four independent 1038 experiments) (e) Histogram showing the distribution of nuclei/cell for the indicated $D$. 1039 discoideum strains (Ax3, $\Delta$ sey1). Cells were stained with DAPI (4',6-diamidino-21040 phenylindole; blue). $32.7 \%$ of $\Delta$ sey 1 cells showed two or more nuclei in contrast to $10.2 \%$ for 1041 Ax3 cells ( $\mathrm{n}=100$ cells). (f) Growth curves of D. discoideum strains cultivated in Petri-dishes 1042 plotted as a function of time (in hours, h). Compared to Ax3 cells, $\Delta$ sey 1 cells show a reduced 1043 growth rate. Doubling times are indicated (data represent the average and s.e.m. of three 1044 independent experiments). (g) Aggregation and development of Ax3 and $\Delta$ seyl cells plated 1045 on solid starvation plates and incubated at $22^{\circ} \mathrm{C}$. Cellular aggregates were observed after $6 \mathrm{~h}$ 1046 and fruiting bodies after $24 \mathrm{~h}$ for both cell lines. Scale bar, $0.5 \mathrm{~mm}$

1047

1048 FIGURE 2 D. discoideum $\Delta$ seyl shows aberrant ER morphology and dynamics. 
1049 (a) ER morphology in Ax3 and $\Delta$ seyl cells analyzed by confocal microscopy after 1050 immunolabeling of the ER-resident enzyme protein disulfide isomerase (PDI). Compared to 1051 the Ax3 parental strain, $\Delta$ seyl cells showed clumps of PDI-positive membranous structures at 1052 the cell periphery and a few interconnected ER tubules (red arrowheads). Nuclei were stained 1053 with DAPI (in blue). Scale bars, $5 \mu \mathrm{m}$. (b) Representative confocal fluorescence micrographs 1054 of dually labeled $D$. discoideum Ax 3 or $\Delta$ seyl mutant cells producing the ER marker calnexin 1055 A-GFP (CnxA-GFP) and the PtdIns(4)P-binding probe P4C-mCherry. Arrowheads, clumped 1056 peripheral ER. Scale bars, $3 \mu \mathrm{m}$. (c) Snapshots of time-lapse video microscopy analysis of 1057 amoebae producing calnexin A-GFP. The ER nodular clumps observed in $\Delta$ seyl cells appear 1058 mostly static (white arrowheads) in contrast to the vivid dynamics of ER tubules in mutant 1059 and parental D. discoideum strains (red arrowheads). N, nucleus. Scale bars, $5 \mu \mathrm{m}$. (d) 1060 Electron micrographs of Ax3 and $\Delta$ sey 1 cells. N, nucleus; arrowheads, rough ER. Scale bars, $10611 \mu \mathrm{m}$.

1062

1063 FIGURE 3 Analysis of endocytic functions and growth on bacteria of $\Delta$ seyl cells.

1064 (a) Fluid-phase uptake of dextran coupled to Alexa-Fluor-647 was measured by flow 1065 cytometry and expressed as the percentage of the maximal uptake in Ax3 cells. (b) Exocytosis 1066 was analyzed in cells preincubated for $4 \mathrm{~h}$ with Alexa-Fluor-647-dextran to fully load all 1067 endocytic compartments. After incubation in dextran-free medium for different periods of 1068 time, retained intracellular fluorescence was measured by flow cytometry and expressed as 1069 the percentage of the initial fluorescence. (c) Representative images of the growth of cells on 1070 lawns of $K$. pneumoniae observed after 5 days $(\mathrm{n}>3)$. $\Delta$ seyl cells were unable to grow on 1071 these bacterial lawns, whereas the production of GFP-Sey1 partially complemented this 1072 defect. (d) Schematic representation of cell capacities to grow on different bacterial lawns. $1073 \Delta$ seyl cells showed total growth inhibition on lawns of Gram-negative K. pneumoniae (K. 
1074 pneum.) and E. coli $\mathrm{B} / \mathrm{r}$ and severely reduced growth rates on lawns of Gram-positive $B$. 1075 subtilis and M. luteus. Cell growth on several bacterial species was assessed as shown in (c) in 1076 three independent experiments and scored from 4 (efficient growth in all tested cell numbers) 1077 to 0 (no growth). Colored squares indicate the average score (green 4, yellow 3, orange 2, and 1078 red 0-1). (e) Uptake of K. pneumoniae was measured by flow cytometry after incubation of 1079 cells with GFP-producing bacteria for $20 \mathrm{~min}$. Results are expressed as the percentage of 1080 uptake in Ax3 cells. (f) Phagocytic rates of Ax3 and $\Delta$ seyl cells incubated with fluorescent 1081 beads. Cells were incubated with $1 \mu \mathrm{m}$ YG-labeled beads, and at the indicated time points, the 1082 internal fluorescence was measured by flow cytometry and expressed as the percent of 1083 maximal uptake by Ax3 cells. (g) Killing of internalized bacteria was measured upon 1084 incubation of $\mathrm{Ax} 3$ or $\Delta$ seyl cells with $K$. pneumoniae. At the indicated time points, samples 1085 were treated with Triton X-100 to selectively lyse amoebae and plated on LB-agar plates. 1086 Bacterial clones were counted after overnight incubation at $37^{\circ} \mathrm{C}$. Data are expressed as the 1087 percent of surviving bacteria. All data in this figure are normalized to protein content in cells 1088 and represent the mean and s.e.m. of three independent experiments (not significant, ns: $P>$ $10890.05, * * P<0.01)$.

1091 FIGURE 4 Hypersecretion of lysosomal enzymes by $\Delta$ seyl cells.

1092 (a) The enzymatic activity of lysozymes in cell lysates was measured by the reduction of 1093 turbidity of heat-killed Micrococcus lysodeikticus in suspension. Results are expressed as the 1094 percentage of activity of strain Ax3 (left panel). When bacteria are included in agarose gels, 1095 halos around diluted cell lysate deposits form proportionally to the extent of bacterial lysis 1096 (right panel) providing a visual estimate of enzymatic activities. (b) $\alpha$-mannosidase and 1097 cathepsin D (CtsD) enzymatic activities were measured in cellular pellets (internal pool) and 1098 extracellular medium (secreted pool) after incubation of the Ax3 and $\Delta$ seyl strains indicated 
1099 in culture medium for $24 \mathrm{~h}$. Results are expressed as the percentage of secreted activities 1100 relatively to total internal and secreted activities. (c) Cathepsin D production by the Ax3 and $1101 \Delta$ seyl strains indicated. Representative samples of cathepsin D (CtsD, $44 \mathrm{kDa}$ ) protein 1102 quantity determined by Western blot of whole cell lysates with an anti-CtsD antibody. 1103 Immunoblotting with an anti-PDI antibody was carried out to control protein amounts of 1104 loaded material. (d) Histogram showing calculated ratio between CtsD and PDI signals in 1105 Western blots quantified using the Image lab analysis software. Results are normalized to the $1106 \mathrm{CtsD} / \mathrm{PDI}$ ratio in Ax3 cells. (e) Intracellular proteolysis was measured by feeding cells with 1107 DQ Green-BSA coated beads and measuring unquenching of the fluorophore after incubation 1108 for $1 \mathrm{~h}$. Proteolysis in $\Delta s e y 1$ cells is expressed as the percentage of Ax3 activity. All data 1109 presented in this figure are the mean and s.e.m. of three independent experiments (not 1110 significant, ns: $P>0.05, * P<0.05, * * P<0.01, * * * * P<0.0001)$.

1112 FIGURE 5 D. discoideum $\Delta$ seyl is impaired for cell motility.

1113 (a) Time-lapse video confocal microscopy of cell motility using single cell tracking analysis.

1114 The indicated Ax3 or $\Delta s e y 1$ strains were incubated in HL5 medium and imaged by spinning 1115 disk confocal microscopy. Randomly moving cells were imaged every $30 \mathrm{~s}$ for 30 min. Tracks 1116 of 15 randomly sampled cells are shown centered at the origin. The motility defect of $\Delta$ seyl 1117 cells was restored upon ectopic production of GFP-Sey1 but not of the catalytically inactive 1118 Sey1_K154A mutant. (b) Quantification of the maximum distance from the origin of 1119 individual cells of each cell line. The median is indicated by a line in each box extending from 1120 the upper and lower quartiles (not significant, ns: $P>0.05$, *** $P<0.001$ ).

1122 FIGURE 6 Analysis of the UPR pathway in D. discoideum $\Delta$ seyl. 
1123 (a) Histogram of cell viability after addition of $1.5 \mu \mathrm{g} / \mathrm{mL}$ tunicamycin (TN) for the indicated 1124 period of times. Viability was measured by flow cytometry analysis as exclusion of propidium 1125 iodide. Results are the mean and s.e.m. of three independent experiments (not significant, ns: $\left.1126 P>0.05,{ }^{* *} P<0.01,{ }^{* * *} P<0.001\right)$. (b) The indicated cell lines were incubated with 1.5 $1127 \mu \mathrm{g} / \mathrm{mL}$ tunicamycin $(\mathrm{TN})$ and observed by phase contrast microscopy after $48 \mathrm{~h}$ of incubation. 1128 Dead cells are indicated by red arrows. Scale bars, $10 \mu \mathrm{m}$. (c) Effect of the TN concentration 1129 on cell viability after $48 \mathrm{~h}$ of incubation. Cell viability was determined as above. (d) 1130 Representative Western blot analysis of $\mathrm{CdcD}$ and PDI protein production in cells after 1131 addition of $1.5 \mu \mathrm{g} / \mathrm{mL}$ TN for $24 \mathrm{~h}$. (e) Quantification of signals in Western blots for CdcD 1132 and PDI bands. Data correspond to the quotient of CdcD or PDI divided by EHD signals and 1133 normalized to corresponding signals in Ax3. Results are the mean and s.e.m. of three and five 1134 independent experiments for $\mathrm{CdcD}$ and PDI, respectively (not significant, ns: $P>0.05, * P<$ $11350.05, * * P<0.01, * * * * P<0.0001)$.

1137 FIGURE 7 D. discoideum $\Delta$ seyl is defective for intracellular replication of L. pneumophila 1138 and rough ER recruitment.

1139 (a) D. discoideum Ax3 or $\Delta$ seyl producing GFP (pDM317) were infected (MOI 1) with 1140 (filled symbols) mCherry-producing L. pneumophila JR32 or (empty symbols) $\Delta i c m T$ 1141 (pNP102) for 6 days. Intracellular replication of the bacteria was assessed by colony-forming 1142 units (CFU), of which the number of CFU per mL was calculated and plotted. Data represent 1143 mean \pm SD of two independent experiments in technical triplicates $(* * * P<0.001)$. (b) $D$. 1144 discoideum Ax3 or $\Delta$ sey1 producing GFP (pDM317) were infected (MOI 50) with mCherry1145 producing L. pneumophila $\Delta i c m T$ (pNP102) for the time indicated. Percentage of surviving 1146 bacteria was derived from CFU. Data represent mean \pm SD of two independent experiments 1147 in technical triplicates (not significant, ns: $P>0.05$ ). (c) D. discoideum Ax3 or $\Delta$ sey 1 
1148 producing GFP (pDM317) or $\Delta$ seyl expressing GFP-Sey1 (pBS001) were infected (MOI 10)

1149 for $2 \mathrm{~h}$ or $8 \mathrm{~h}$ with mCherry-producing L. pneumophila JR32 (upper panels) or $\Delta i c m T$ (lower

1150 panels). Samples were fixed, stained with uranyl acetate, embedded in Epon/Araldite and

1151 analyzed by electron microscopy. Star: L. pneumophila; arrowhead: rough endoplasmic 1152 reticulum (ER). Scale bars, $500 \mathrm{~nm}$.

1153

1154 FIGURE 8 D. discoideum $\Delta$ seyl cells are impaired for LCV expansion.

1155 (a) Representative confocal fluorescence micrographs of dually labeled D. discoideum Ax3 1156 (upper panel) or $\Delta s e y l$ mutant (lower panel) producing calnexin-GFP (CnxA-GFP) and P4C1157 mCherry, infected (MOI 5, 2 h) with mCerulean-producing L. pneumophila JR32 (pNP99). 1158 Scale bars, $3 \mu \mathrm{m}$. (b) Imaging flow cytometry (IFC) analysis of dually labelled $D$. discoideum $1159 \mathrm{Ax} 3$ and $\Delta$ seyl mutant producing calnexin-GFP (CnxA-GFP) and P4C-mCherry, infected 1160 (MOI 5) with mPlum-producing L. pneumophila JR32 (pAW014). Representative images at 6 1161 h p.i. are depicted; scale bars, $7 \mu \mathrm{m}$ (top panel). Quantification of CnxA-GFP or P4C1162 mCherry localizing to LCVs during early time points of infection (bottom panel). Number of 1163 events per sample, $n=111-1565$. Data represent mean \pm SD of three independent experiments $1164(* P<0.05, * * * P<0.001)$. (c) Representative confocal fluorescence micrographs of dually 1165 labeled D. discoideum Ax3 (upper panels) or $\Delta$ sey 1 mutant (lower panels) producing CnxA1166 GFP and P4C-mCherry, infected (MOI 5) with mCerulean-producing L. pneumophila JR32 1167 (pNP99) for $2 \mathrm{~h}, 8 \mathrm{~h}, 16 \mathrm{~h}$ or $24 \mathrm{~h}$. Scale bars, $3 \mu \mathrm{m}$. (d) Quantification of (C) LCV area at the 1168 analyzed time points $(n=100)$. Data represent mean $\pm 95 \%$ CI of three independent 1169 experiments $(* * * P<0.001)$. 


\section{SUPPORTING INFORMATION}

1172 FIGURE S1 Confocal microscopy analysis of the ER morphology upon ectopic production 1173 of GFP-Sey1, GFP-Sey1_K154A or GFP-Rtnlc.

1174

1175 FIGURE S2 Confocal microscopy analysis of Sec22b, Ufe1 and Use1 localization.

1177 FIGURE S3 Confocal microscopy analysis of intracellular compartments and microtubule 1178 architecture.

1179

FIGURE S4 Confocal microscopy analysis of PtdIns(3) $P$ and PtdIns(4) $P$ localization and 1181 actin protrusions.

1182

1183 FIGURE S5 Analysis of lysosomal enzyme secretion, carbohydrate processing and 1184 acidification of endocytic compartments.

1185

1186 FIGURE S6 $D$. discoideum $\Delta$ seyl cells are defective for intracellular replication of $L$. 1187 pneumophila and LCV expansion.

1189 FIGURE S7 Schematic representation of the strategies followed for genetic inactivation of 1190 seyl or rtnlc, and PCR-based validation.

1192 TABLE S1 Strains and plasmids used in this study.

1194 TABLE S2 Oligonucleotides used in this study. 
1196 TABLE S3 Antibodies used in this study.

1197

1198 MOVIE S1 Time-lapse video microscopy analysis of Ax3 cells producing the ER marker, 1199 GFP-calnexin. Images were taken every second for $2 \mathrm{~min}$.

1200

1201 MOVIE S2 Time-lapse video microscopy analysis of $\Delta s e y 1$ cells producing the ER marker, 1202 GFP-calnexin. Images were taken every second for $2 \mathrm{~min}$.

1203

1204 MOVIE S3 Time-lapse video microscopy analysis of Ax3 cells producing the cytoskeleton 1205 protein tubulin A, GFP-TubA. Images were taken every second for $2 \mathrm{~min}$.

1206

1207 MOVIE S4 Time-lapse video microscopy analysis of $\Delta$ seyl cells producing the cytoskeleton 1208 protein tubulin A, GFP-TubA. Images were taken every second for 2 min. 
(a)

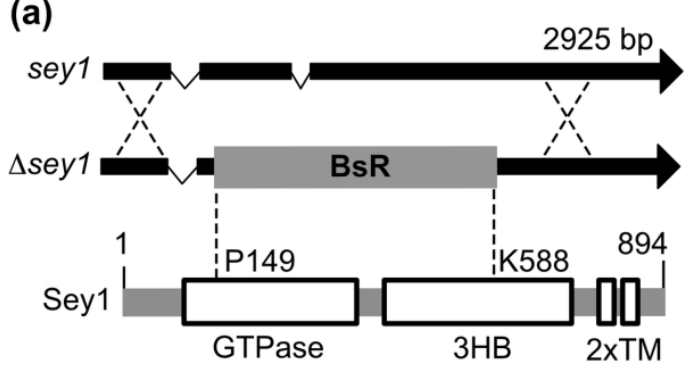

(c)

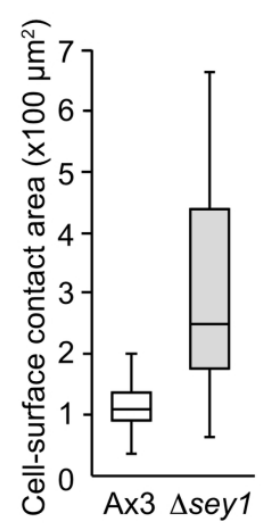

(d)

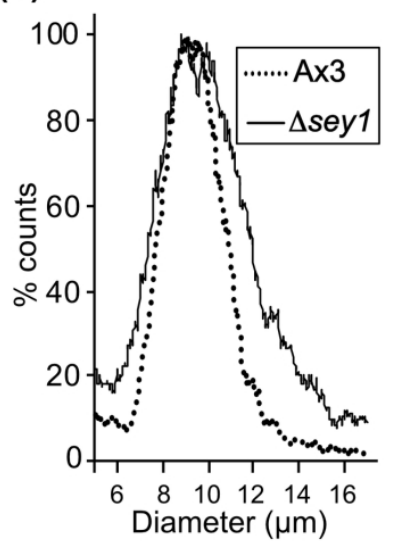

(b)

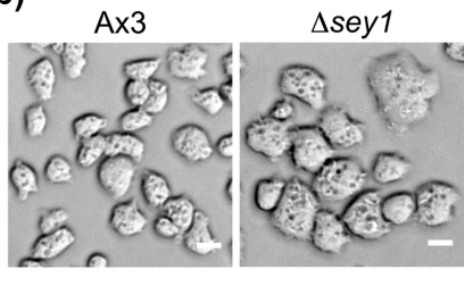

(e)

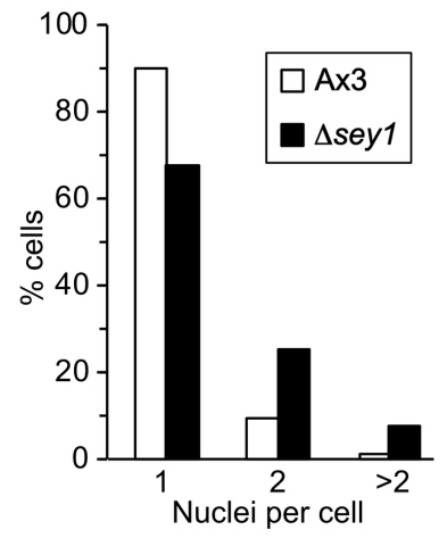

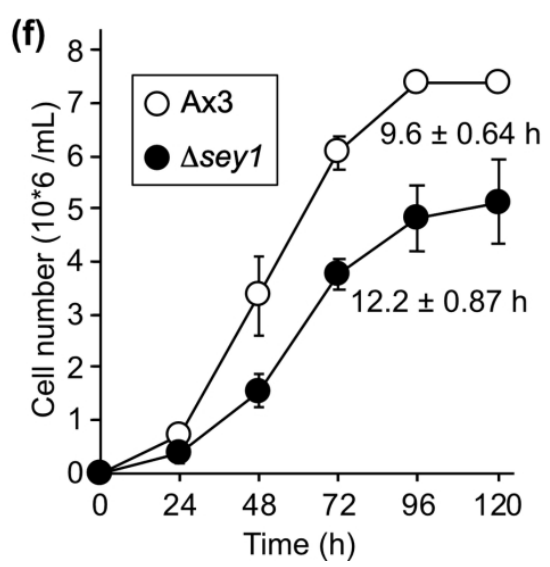

(g)

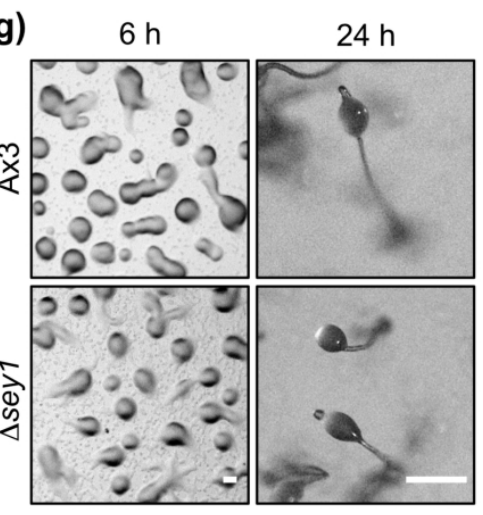

Figure 1

$162 \times 215 \mathrm{~mm}(300 \times 300 \mathrm{DPI})$ 
(a)

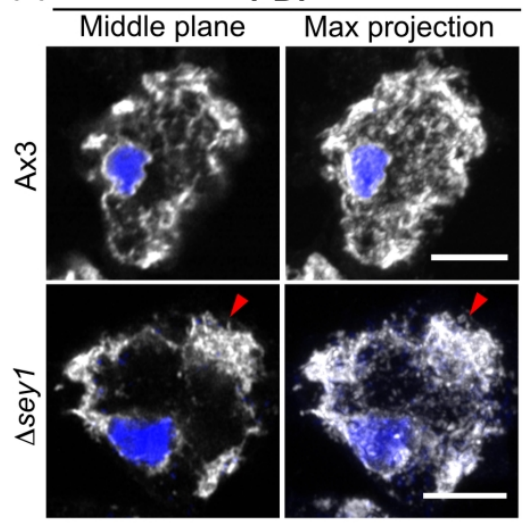

(b)

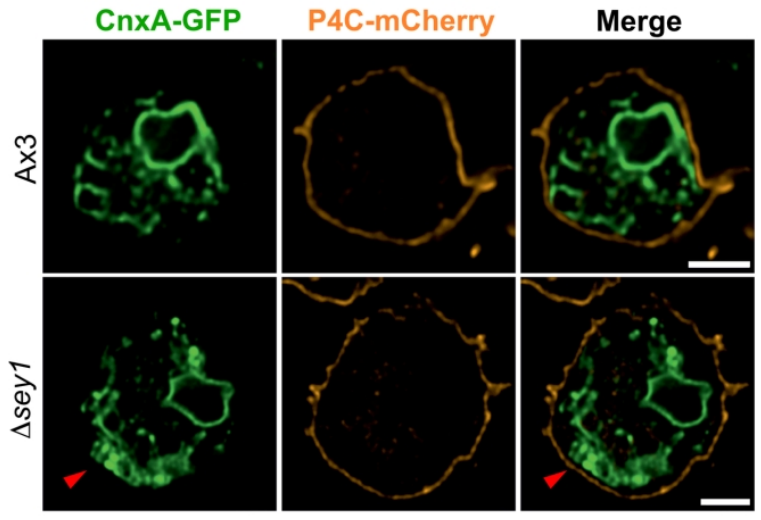

(c)
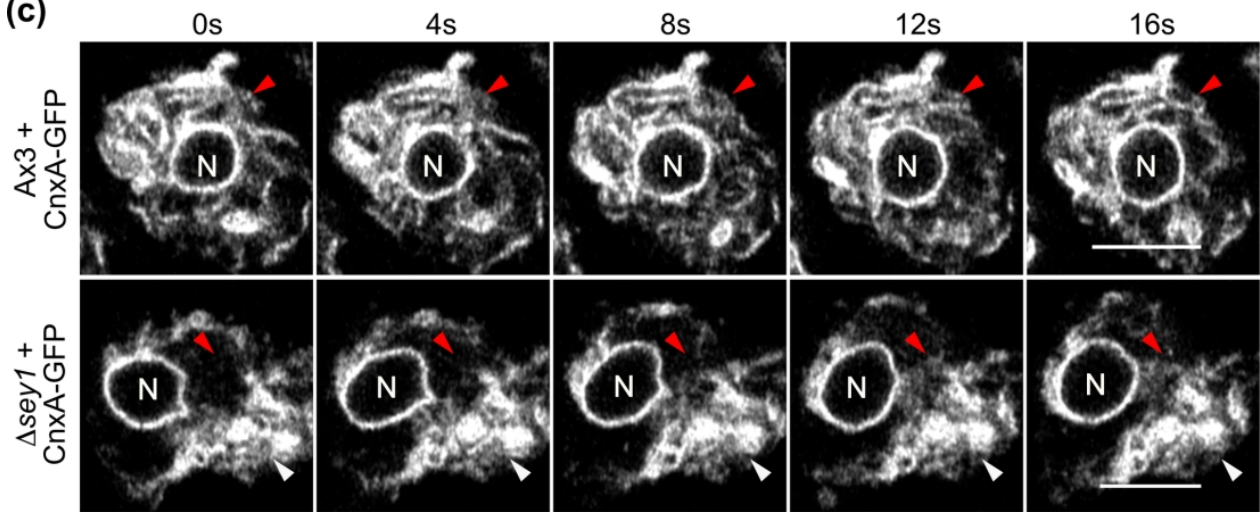

(d)

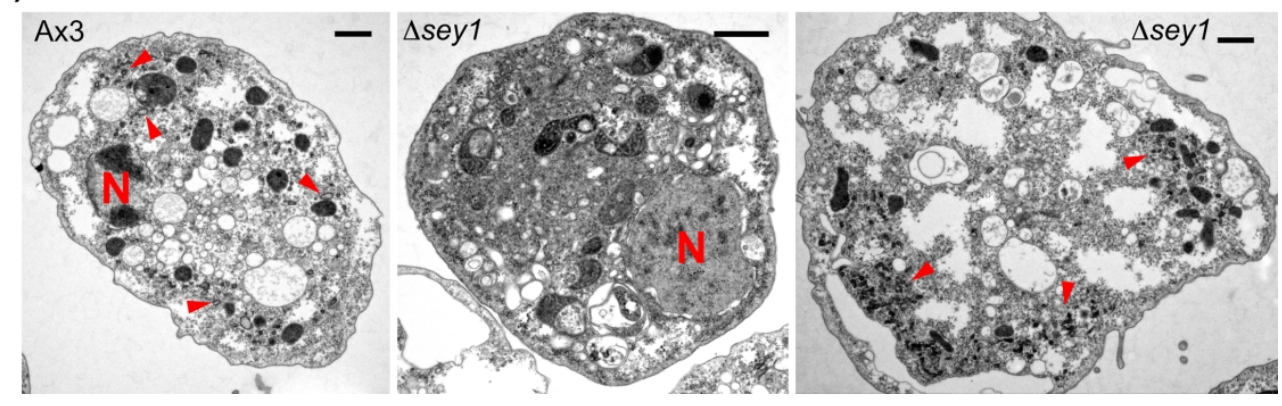

Figure 2

$186 \times 225 \mathrm{~mm}(300 \times 300$ DPI $)$ 
(a)

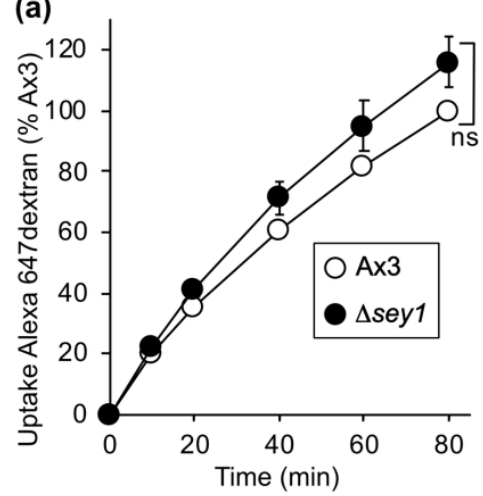

(c)

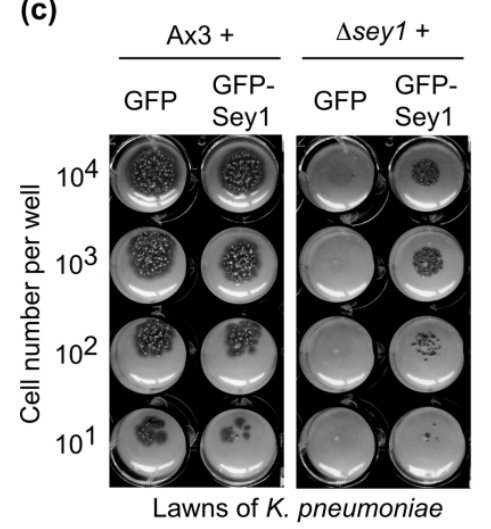

(f)

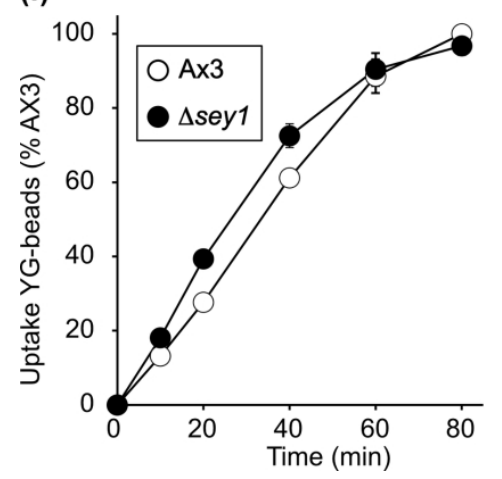

(b)

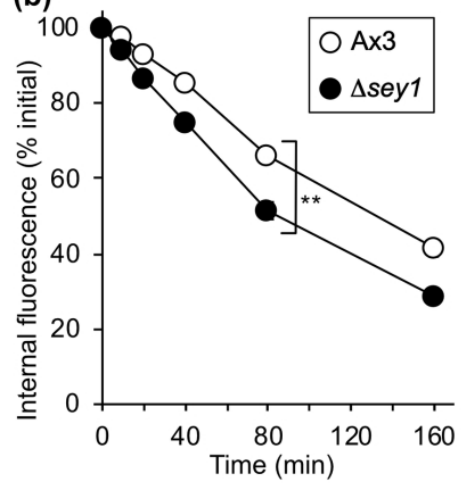

(d)

(e)

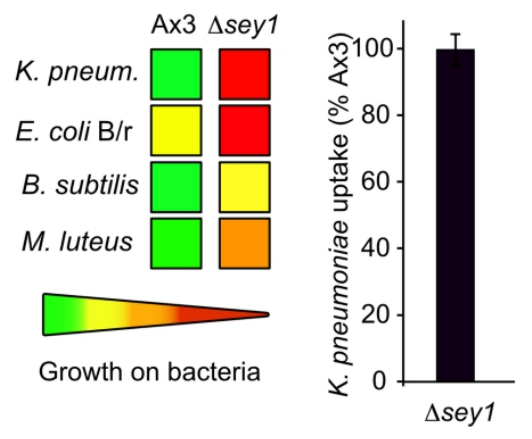

(g)

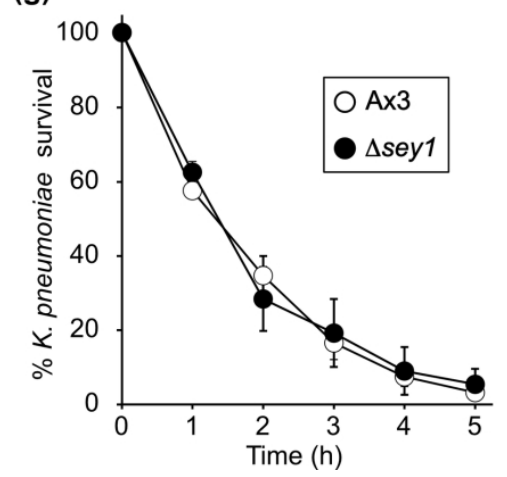

Figure 3

$162 \times 250 \mathrm{~mm}(300 \times 300$ DPI $)$ 
(a)

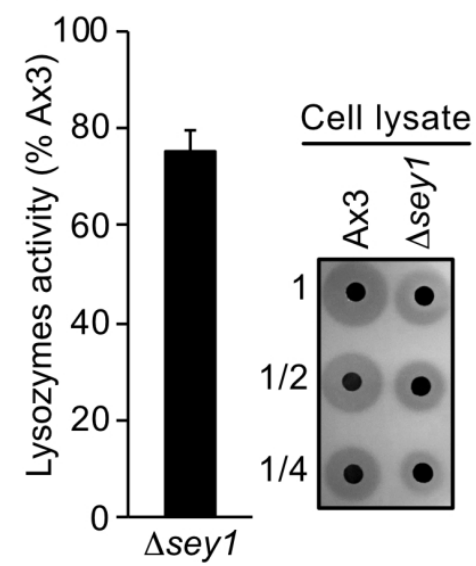

(b) Cathepsin D

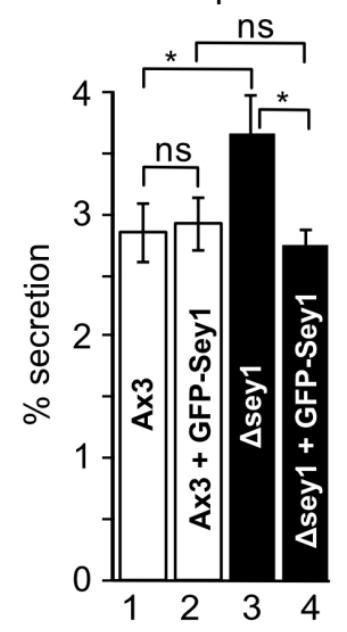

(c)

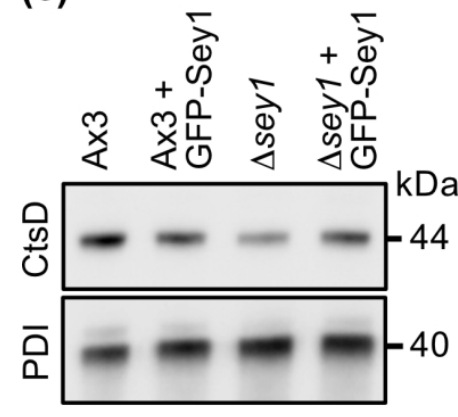

(d)

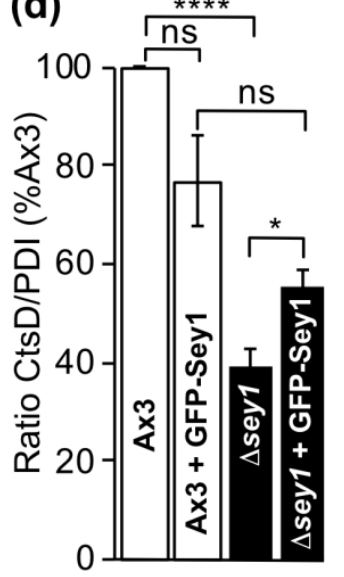

Alpha-

mannosidase

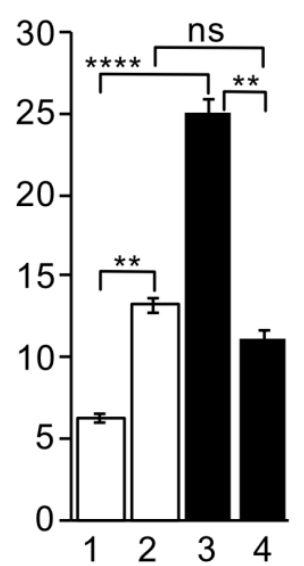

(e)

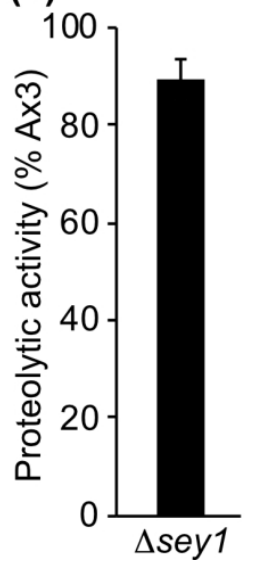

Figure 4 
(a)

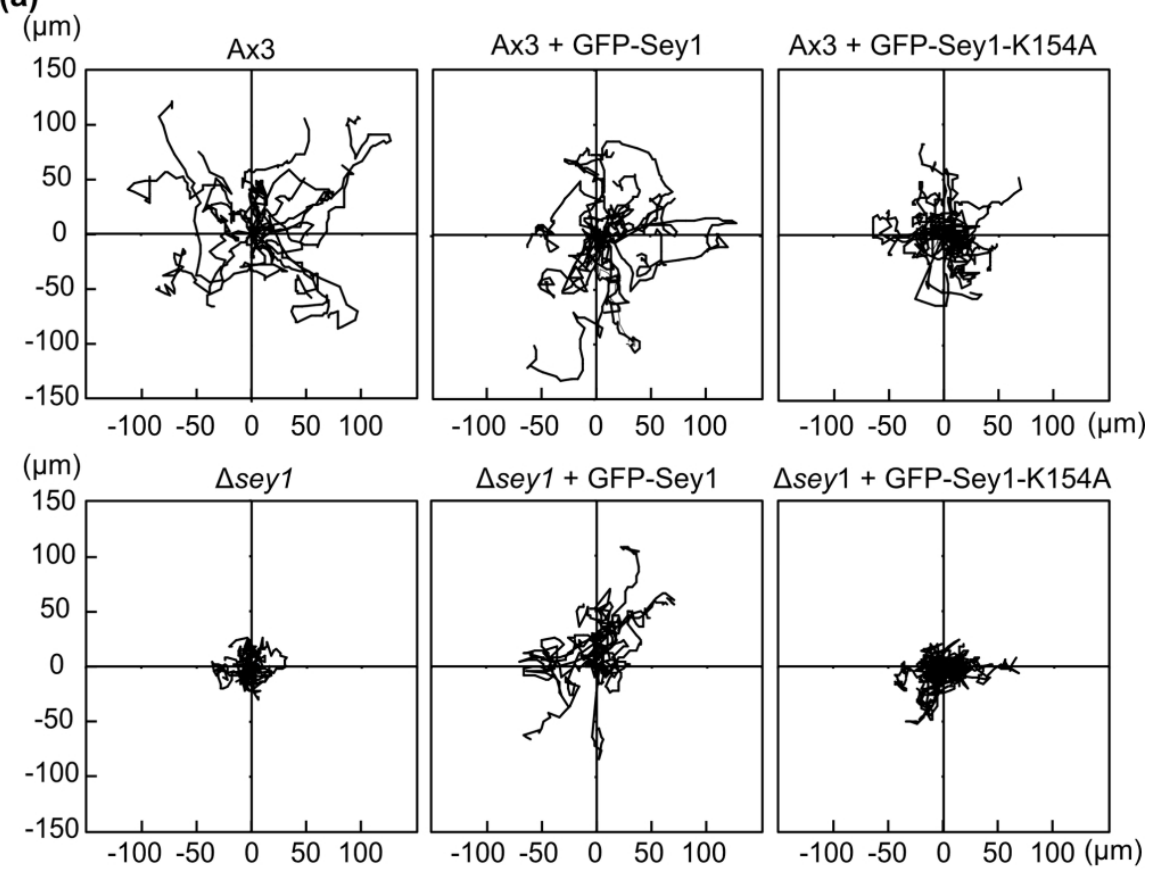

(b)

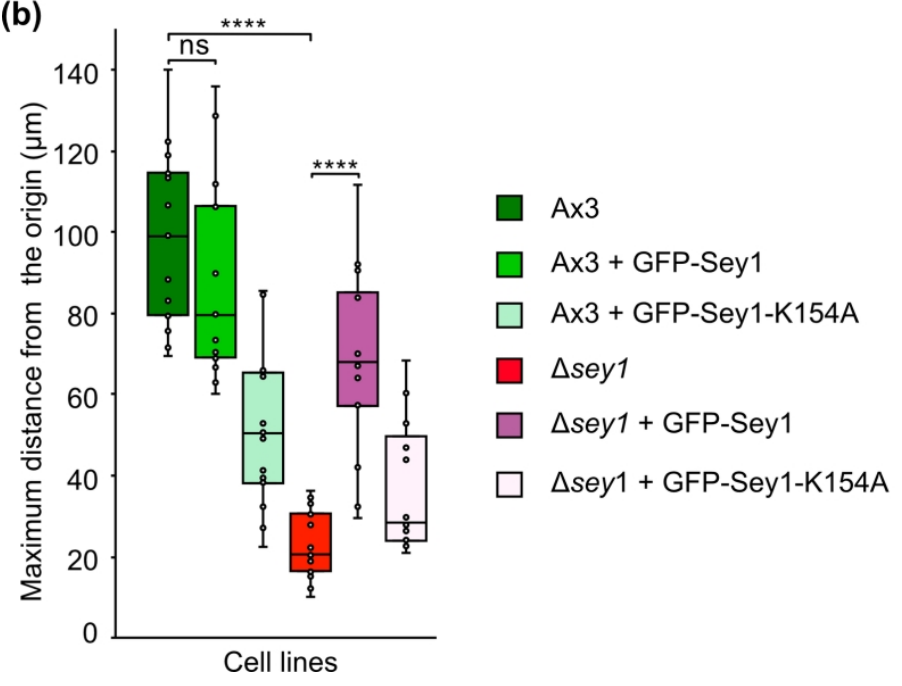

Figure 5

$171 \times 233 \mathrm{~mm}(300 \times 300$ DPI $)$ 


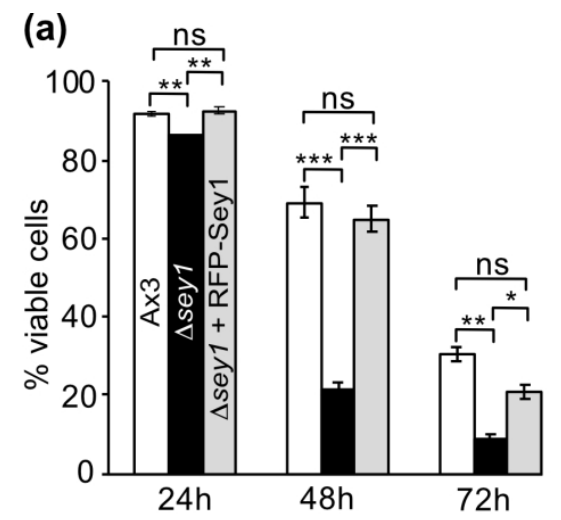

(b)

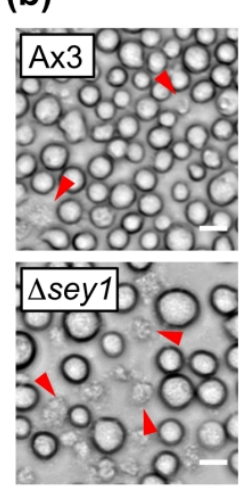

(e)

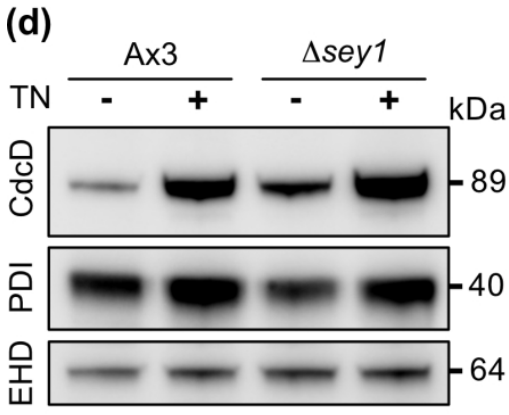

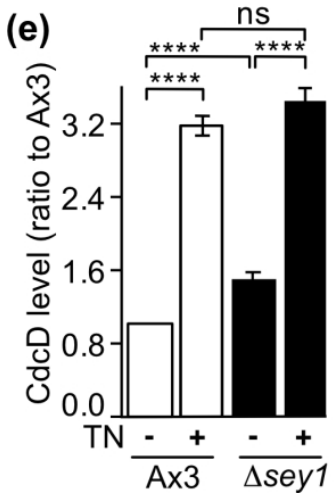

(c)
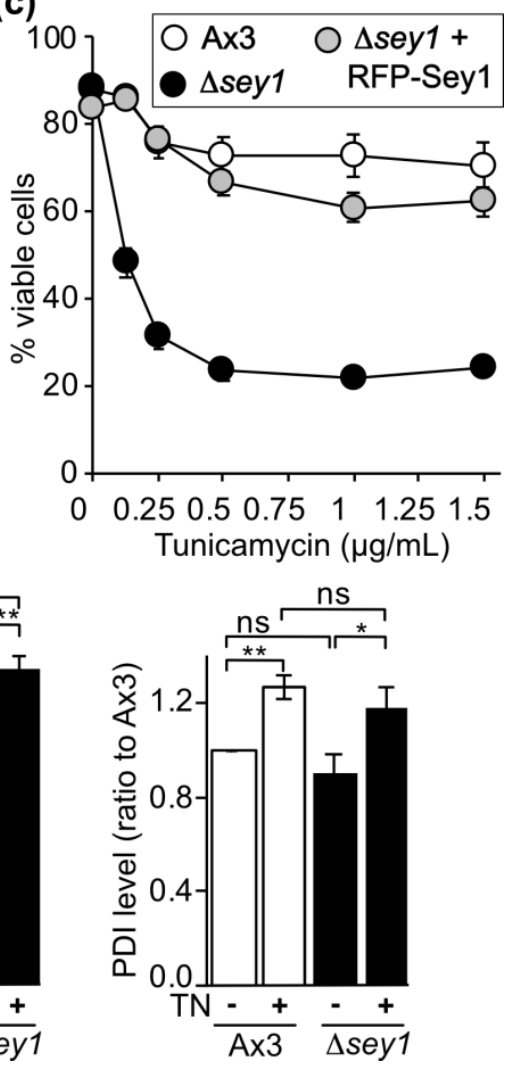

Figure 6 
(a)

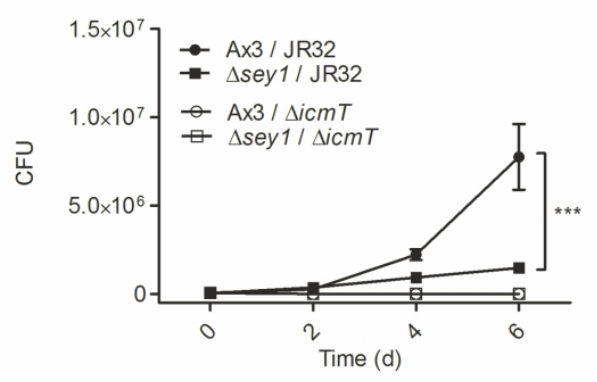

(b)

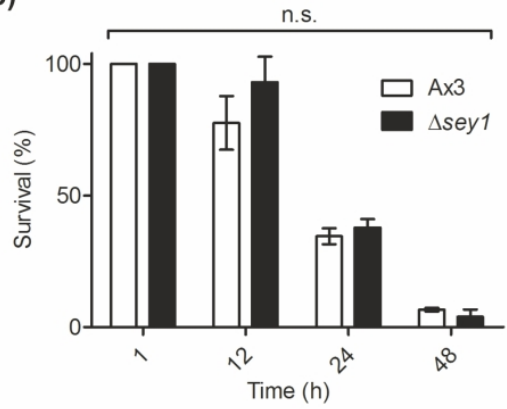

(c)
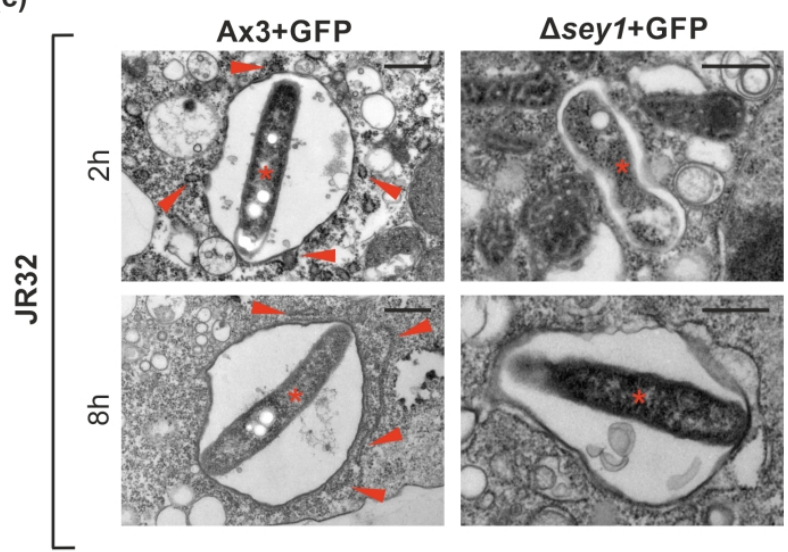

Dsey1+GFP-Sey1
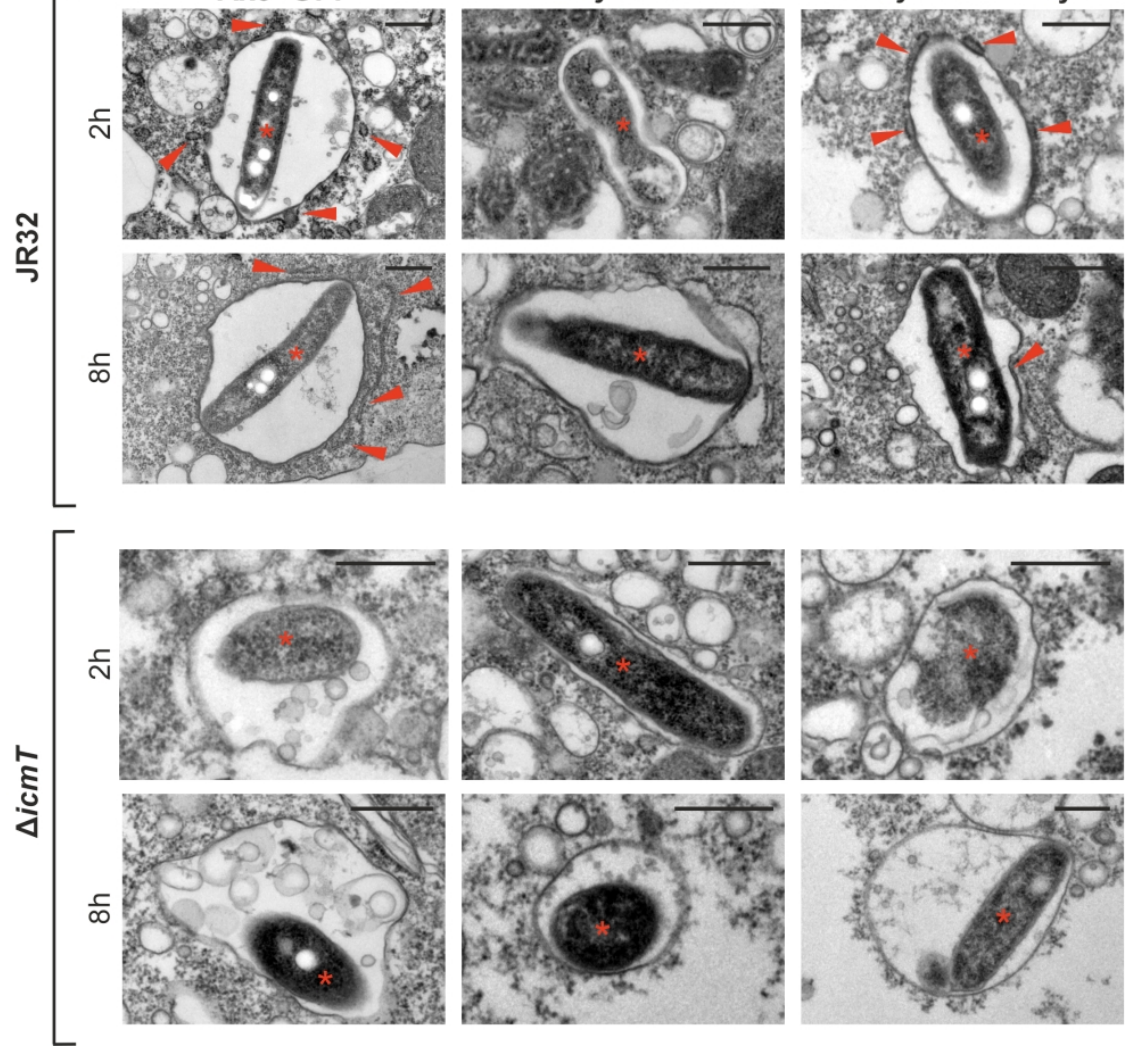

Figure 7 
(a)

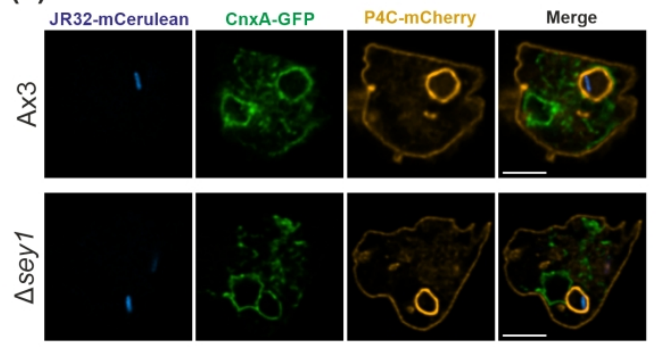

(b)
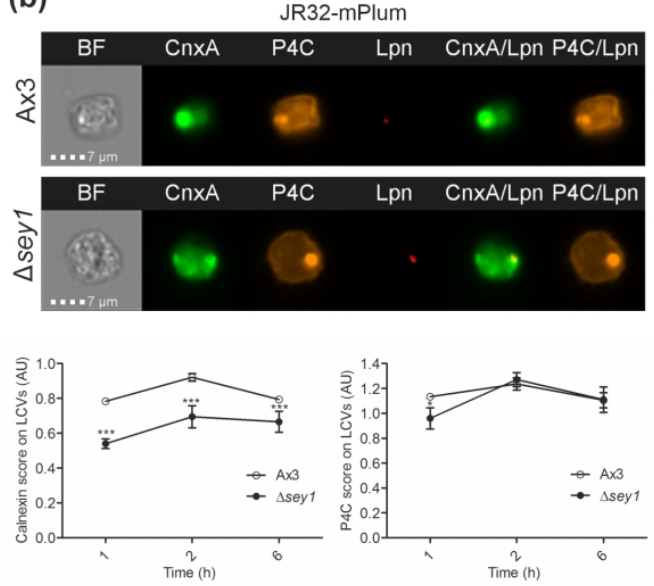

(d)

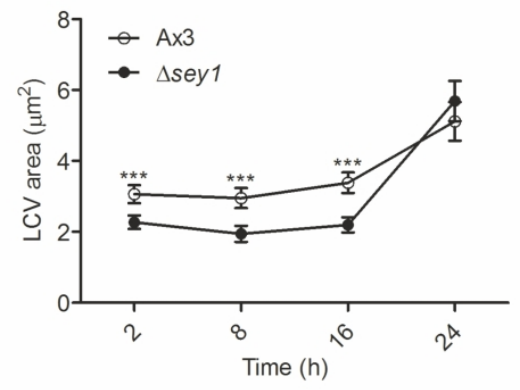

(c)
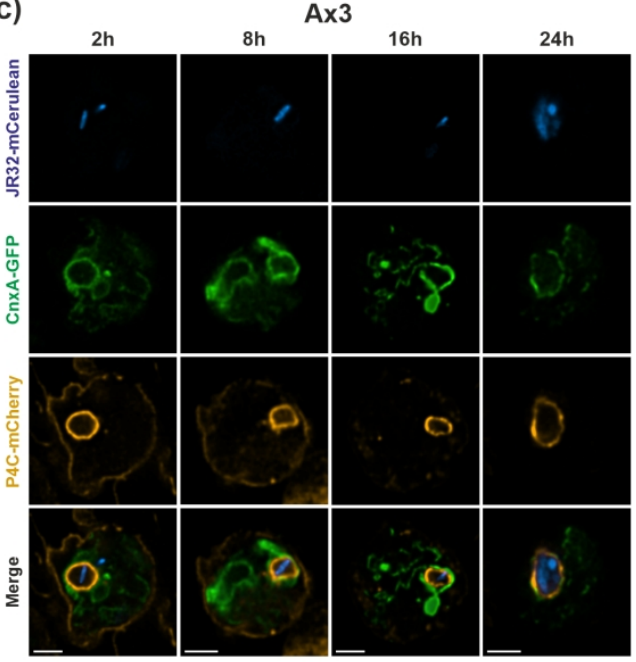

$\Delta$ sey1

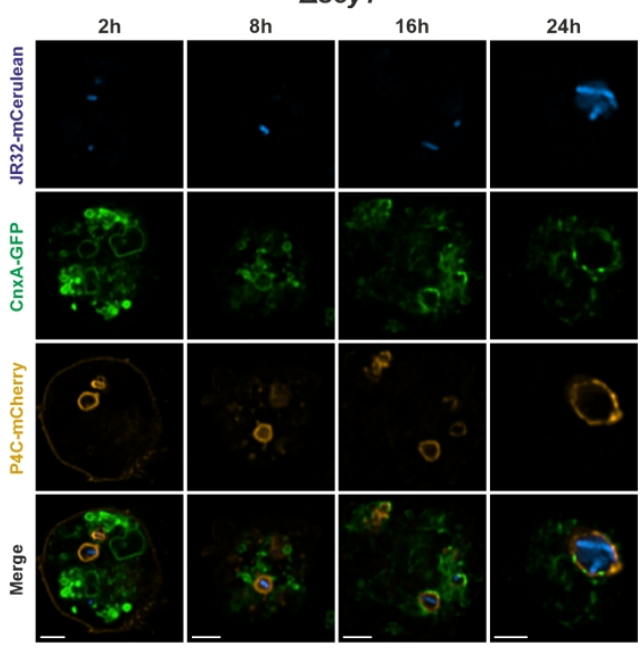

Figure 8 